\title{
ZBTB20 Positively Regulates Oxidative Stress, Mitochondrial Fission, and Inflammatory Responses of ox-LDL-Induced Macrophages in Atherosclerosis
}

\author{
Jun Tao, ${ }^{1,2}$ Junxiong Qiu, ${ }^{1,2}$ Liuyi Lu, ${ }^{1,2}$ Lisui Zhang, ${ }^{2}$ Yuan Fu, ${ }^{2}$ Meng Wang, ${ }^{2}$ Jingjun Han, ${ }^{3}$ \\ Maomao Shi, ${ }^{2}$ Ling Li, ${ }^{2}$ Zongkai Zhao, ${ }^{2}$ Feng Wei, ${ }^{2}$ Chao Wang, ${ }^{2}$ Haifeng Zhang $\mathbb{D}^{4,5}$ \\ Shi Liang $\mathbb{D}$, 1,2 and Junmeng Zheng $\mathbb{D}^{1,2}$ \\ ${ }^{1}$ Guangdong Provincial Key Laboratory of Malignant Tumor Epigenetics and Gene Regulation, Sun Yat-sen Memorial Hospital, \\ Sun Yat-sen University, Guangzhou, China 510120 \\ ${ }^{2}$ Department of Cardiovascular Surgery, Sun Yat-sen Memorial Hospital, Sun Yat-sen University, Guangzhou, China 510120 \\ ${ }^{3}$ Department of Thoracic and Cardiac Surgery, The Eighth Affiliated Hospital, Sun Yat-sen University, Shenzhen, China 518033 \\ ${ }^{4}$ Department of Cardiology, Sun Yat-sen Memorial Hospital, Sun Yat-sen University, Guangzhou, China 510120 \\ ${ }^{5}$ Laboratory of Cardiac Electrophysiology and Arrhythmia in Guangdong Province, Guangzhou, China 510120
}

Correspondence should be addressed to Haifeng Zhang; zhanghf9@mail.sysu.edu.cn, Shi Liang; md02ls@mail2.sysu.edu.cn, and Junmeng Zheng; zhengjm27@mail.sysu.edu.cn

Received 6 January 2021; Revised 26 January 2021; Accepted 9 February 2021; Published 11 March 2021

Academic Editor: Yun-dai Chen

Copyright (C) 2021 Jun Tao et al. This is an open access article distributed under the Creative Commons Attribution License, which permits unrestricted use, distribution, and reproduction in any medium, provided the original work is properly cited.

\begin{abstract}
Atherosclerosis (AS) is one of the most serious and common cardiovascular diseases affecting human health. AS is featured by the accumulation of plaques in vessel walls. The pathophysiology of AS is relevant in the low-density lipoprotein (LDL) uptake by macrophages, as well as the conversion of macrophages to foam cells. However, the mechanisms about how macrophages regulate AS have not been fully elucidated. In this study, we aimed to illuminate the roles of ZBTB20 and to excavate the underlying regulative mechanisms of ZBTB20 in AS. The microarray analysis revealed that ZBTB20 was a hub gene in the oxidative stress and inflammatory responses induced by oxidized LDL (ox-LDL) in AS. Correspondingly, our validation studies showed that ZBTB20 increased in either the human atherosclerotic lesion or the ox-LDL-stimulated macrophages. Moreover, the knockdown of ZBTB20 decreased M1 polarization, suppressed the proinflammatory factors, inhibited mitochondrial fission, and reduced the oxidative stress level of macrophages induced by ox-LDL. The mechanistic studies revealed that the ZBTB20 knockdown suppressed NF- $\kappa \mathrm{B} / \mathrm{MAPK}$ activation and attenuated the mitochondrial fission possibly via regulating the nucleus translocation of NRF2, a pivotal transcription factor on redox homeostasis. Our in vivo studies showed that the sh-ZBTB20 adenovirus injection could reduce the progression of AS in apolipoprotein E-deficient (ApoE ${ }^{-/}$) mice. All in all, these results suggested that ZBTB20 positively regulated the oxidative stress level, mitochondrial fission, and inflammatory responses of macrophages induced by ox-LDL, and the knockdown of ZBTB20 could attenuate the development of AS in ApoE ${ }^{-/-}$mice. $^{-}$
\end{abstract}

\section{Introduction}

AS is a chronic vascular disease featured by the accumulation of plaques within the vessel wall of the large- and middlesized arteries $[1,2]$. Up to date, various factors including hypertension, diabetes mellitus, high cholesterol level, smoking, and adiposity have been found to be associated with the development of AS $[3,4]$. The pathophysiology of AS is closely related to the LDL uptake by macrophages and subsequent differentiation into foam cells. The plaques formed by macrophages have different subtypes based on the activation stimuli and protein expression patterns. Thus, macrophages may exert either harmful effects or beneficial effects in the progression of AS $[5,6]$. Recent studies have proposed that targeting of the macrophage may be a crucial target to ameliorate vulnerable plaques and subsequently alleviate AS [7]. 
However, the underlying mechanistic role of macrophages in the pathophysiology remains unclear. Hence, it is of paramount importance to further elucidate the underlying mechanisms of macrophage-mediated AS, which may provide novel therapies for the treatment.

Recently, studies demonstrated that mitochondria not only can regulate innate immune responses [8] but also can modulate the level of reactive oxygen species (ROS) to affect the homeostasis and inflammatory status of macrophages [9]. There is growing evidence showing that AS may be associated with the dysregulated mitochondrial function and bioenergetics [10]. Chen et al. demonstrated that CD36 signaling regulated mitochondrial metabolic reprogramming, which subsequently drives macrophage inflammatory responses in AS [11]. Besides, Dicer in macrophages was found to prevent AS by promoting mitochondrial oxidative metabolism [12]. Recently, myeloperoxidase-derived oxidant hypothiocyanous acid (HOSCN) was found to induce mitochondrial dysfunction in macrophages, which may be associated with the pathophysiology of AS [13]. Xin et al. also demonstrated that ox-LDL activates the dynamin-related protein 1 (DRP1) level as well as the mitochondrial fission status of macrophages [14].

ZBTB20, mainly known as a transcriptional repressor, is a member of the POZ and Krüppel family, with a zinc finger domain and an intact BTB domain $[15,16]$. Up to date, the diverse functions of ZBTB20 have been reported, and studies demonstrated that ZBTB20 could regulate ion channels, remodeling, immunity, and inflammation [17-20]. In vascular diseases, especially in the development of AS, NF- $\kappa$ B signaling functions as a key modulator in AS plaque initiation and evolution [21]. Emerging evidence suggests that the NF- $\kappa$ B pathway was activated during the transformation of cholesterol-rich foam cells after taking in LDL, as well as the oxidation of LDL [22]. As for ZBTB20, in vascular diseases, it can regulate cardiac remodeling after myocardial infarction via ROS/TNF- $\alpha$ signaling [23]. Recently, a study by Liu et al. showed that ZBTB20 was able to inhibit the transcription of the $I \kappa B \alpha$ gene, which is a key element in NF- $\kappa \mathrm{B}$ signaling. Our study has also found that during the macrophage-mediated osteolysis, ZBTB20 could adjust the inflammatory response and polarization of macrophages via regulating $I \kappa B \alpha$ transcription and NF- $\kappa$ B activation [18]. However, the roles of macrophage ZBTB20 during the progression of AS remain to be examined.

Here, we examined the expression of ZBTB20 in the macrophages stimulated by ox-LDL and the human AS lesions. Furthermore, the loss-of-function studies were carried out to determine the roles of ZBTB20 on the inflammatory responses, oxidative stress, and mitochondrial fission of the ox-LDLstimulated macrophages. Besides, the subsequent signaling pathways were also examined. Finally, the effects of ZBTB20 on the AS progression were evaluated in the $\mathrm{ApoE}^{-/-}$mice. In brief, the present study may provide novel insights into the roles of ZBTB20 in the pathophysiology and progression of AS.

\section{Materials and Methods}

2.1. Clinical Samples. Clinical samples were collected from 16 patients, including 9 males and 7 females (average age: 57.1 \pm 13.1 years). These patients have accepted bypass operation of the coronary artery because of coronary diseases from 2017 to 2019. Those coronary artery tissues containing AS were collected. The internal mammary artery tissues without AS were used as the control group. All procedures were carried out with the approval of the Ethics Committee of Sun Yat-sen University, Sun Yat-sen Memorial Hospital (SYSEC-KY-KS2020-090).

2.2. Cell Lines and Cell Culture. The macrophages, RAW264.7, were from Procell Life Science \& Technology. The RAW264.7 macrophages were cultured in high-glucose DMEM, containing 10\% fetal bovine serum (FBS) [24]. Cells were cultured at $37^{\circ} \mathrm{C}$ and $5 \% \mathrm{CO}_{2}$. The macrophages were seeded $24 \mathrm{~h}$ before the experiments.

2.3. Small Interfering RNA (siRNA), Cell Transfections, and Treatments. The siRNA targeting ZBTB20 was synthesized by the RiboBio company (Guangzhou, China), and the scrambled siRNA was served as the negative control (NC). For the cell transfections, the ZBTB20-siRNA or the scrambled siRNA was transfected into the macrophages with RNAiMAX (Thermo Fisher Scientific) [25]. Forty-eight hours later, the transfected macrophages were collected for further experimentation.

The ox-LDL was from Yeasen Biotech Co., Ltd., and a concentration of $50 \mu \mathrm{g} / \mathrm{ml}$ was adopted for respective time durations. After that, the macrophages were subjected to further experimental assays. The NRF2 inhibitor (ML385) was purchased from Selleck, and a concentration/duration of $5 \mu \mathrm{M} / 24 \mathrm{~h}$ was used before further experimental assays [26].

2.4. Oil Red O Staining. After treatment, the RAW264.7 macrophages were treated with $4 \%$ PFA for $15 \mathrm{~min}$ and stained with oil red $\mathrm{O}$ solution for $60 \mathrm{~min}$. For the mouse aortas, the adipose tissues were stripped from the aortas [27]. After washing with PBS three times, the aortas were stained with oil red $\mathrm{O}$ solution for $60 \mathrm{~min}$. The stained macrophages and tissues were imaged using a biomicroscope (DM2000, Leica).

2.5. Dil-ox-LDL Uptake of Macrophages. The macrophages were incubated with red fluorescence-labeled Dil-ox-LDL $\left(50 \mu \mathrm{g} / \mathrm{ml}\right.$; Yeasen Biotech Co., Ltd.) for $24 \mathrm{~h}$ at $37^{\circ} \mathrm{C}$ [28]. After that, the macrophages were washed by PBS three times, and a biomicroscope (DM2000, Leica) was used.

2.6. PPI Network Construction and Identification of Hub Genes. A microarray data of the GSE54666 dataset was obtained from the GEO database. The STRING database and Cytoscape software were used to construct a protein-protein interaction network (PPIN) of differentially expressed genes (DEGs). The topology property of the network was analyzed using the MCODE application of Cytoscape software. The functional clustering of the DEGs was performed using the Metascape online tool (https://metascape.org).

2.7. RNA Extraction and $q R T-P C R$. RNAiso Plus (TaKaRa) was used to collect the RNA of RAW264.7 macrophages. A NanoDrop instrument was used to measure the concentrations of RNA [29]. After that, cDNA was obtained by reversetranscribing RNA with PrimeScript RT Mix (TaKaRa). Then, 
qRT-PCR was carried out on a Roche Real-Time PCR System using SYBR Green Mix (Yeasen Biotech Co., Ltd.). Table 1 shows the primers used.

2.8. Western Blot Assay and ELISA. The total protein from RAW264.7 macrophages was obtained by using the RIPA buffer (Beyotime). A nuclear and cytoplasmic extraction kit (CWbiotech) was used to, respectively, obtain the cytosolic protein and nuclear protein. A total of $30 \mu \mathrm{g}$ protein in each lane was prepared, followed by separating in a $10 \%$ polyacrylamide SDS-PAGE gel [30]. After transferring, the PVDF membranes were then blocked with 5\% BSA, then incubated with different antibodies including NRF2, Histone H3, pJNK, KEAP1, DRP1, p-ERK, FIS1, ZBTB20, p-p65, I $\kappa$ B $\alpha$, pp38, IRF3, p-IRF3, and GAPDH (Cell Signaling Technology) [31]. After the incubation, a secondary antibody with linked HRP (Cell Signaling Technology), an ECL detection kit (Yeasen Biotech Co., Ltd.), and a digital imaging system (Kodak) were used.

To detect the cytokines, including TNF- $\alpha$, IL- 6 , and IFN- $\beta$, the macrophage supernatants were collected, followed by detection with ELISA kits (Neobioscience Technology Co., Ltd.).

2.9. Flow Cytometry for Macrophage Polarization and ROS Detection. For the detection of macrophage polarization, iNOS and CD206 were adopted as M1 and M2 polarization markers [32]. Briefly, after incubating in the fixation buffer and washing by the perm/wash buffer, the RAW264.7 macrophages were then incubated in the iNOS antibody or CD206 antibody for $20 \mathrm{~min}$, respectively. After that, these macrophages were washed three times, resuspended in $200 \mu \mathrm{l}$ PBS for each sample, and analyzed with BD Biosciences flow cytometry.

The ROS production was evaluated using a $2^{\prime}, 7^{\prime}$-DHEDA staining kit (KeyGen Biotech). A concentration of $2 \mu \mathrm{M}$ and an incubation duration of $20 \mathrm{~min}$ were adopted [33]. After the incubation, the ROS-positive cell numbers were detected by BD Biosciences flow cytometry, and the fluorescence intensity of the ROS probe was observed on an Olympus fluorescence microscope.

2.10. Mitochondrial Staining of Macrophages. The mitochondrial staining of the macrophages was performed using the MitoTracker Red CMXRos reagent (Beyotime). Briefly, RAW264.7 macrophages after different treatments were incubated with the MitoTracker Red CMXRos reagent for $30 \mathrm{~min}$ [34]. Then, the cells were counterstained with DAPI for $10 \mathrm{~min}$ and imaged under an Olympus fluorescence microscope.

2.11. In Vivo Animal Models of Atherosclerosis and Atherosclerotic Lesion Analysis. Adenovirus expressing shZBTB20 or sh-NC was purchased from GeneChem. The ApoE $^{-/-}$mice (20-30 grams, 8-10 weeks) were purchased from GemPharmatech Co. Ltd. To study the effects of the ZBTB20 knockdown on the AS mouse model, the animals were injected with adenovirus expressing sh-ZBTB20 or sh-NC via the tail, and then the ApoE ${ }^{-/-}$mice were fed with a high-fat diet for 12 weeks. At the end of the experiments [35], the animals were killed by an overdose of pentobarbi-
TABle 1: Primers used in this study.

\begin{tabular}{llc}
\hline ZBTB20 & $\begin{array}{l}\text { Forward } \\
\text { Reverse }\end{array}$ & $\begin{array}{c}\text { GTGGACCGAATCTACTCCGC } \\
\text { CATGAATGCGTGTGATCCAGC }\end{array}$ \\
\hline \multirow{2}{*}{ iNOS } & $\begin{array}{l}\text { Forward } \\
\text { Reverse }\end{array}$ & $\begin{array}{c}\text { GGAGTGACGGCAAACATGACT } \\
\text { TCGATGCACAACTGGGTGAAC }\end{array}$ \\
\hline \multirow{2}{*}{ COX-2 } & Forward & TGCACTATGGTTACAAAAGCTGG \\
& Reverse & TCAGGAAGCTCCTTATTTCCCTT \\
\hline \multirow{2}{*}{ GAPDH } & Forward & TGTGTCCGTCGTGGATCTGA \\
& Reverse & TTGCTGTTGAAGTCGCAGGAG \\
\hline
\end{tabular}

tone $(80 \mathrm{mg} / \mathrm{kg}$, intravenous injection), and the tissues were collected for further experimental assays. All the guidelines of the Institutional Animal Care and Use Committee of Sun Yat-sen University were followed during the animal experiments [36].

The collected tissues were fixed and embedded, then sliced into sections using a microtome. The sections were then dewaxed using the xylene. The H\&E staining kit (Beyotime), CD68 antibody (Servicebio), Masson stain kit (Solarbio), $\alpha$ SMA antibody (Beyotime), and EVG stain kit (Abcam) were used to evaluate the AS lesions [37].

2.12. Immunofluorescent Staining of Macrophages. For the immunofluorescent staining of the macrophages, the macrophages with different treatments were fixed with 4\% PFA, followed by incubation with $0.1 \%$ Triton X-100 and blocking with $1 \%$ BSA. Then, the macrophages were incubated with TNF- $\alpha$, IL-6, p65, IRF3, or NRF2 antibodies (Cell Signaling Technology) overnight at $4^{\circ} \mathrm{C}$ [38]. Alexa Fluor 555 conjugate immunofluorescent secondary antibodies and a Carl Zeiss confocal microscope were used to observe the macrophages.

2.13. Statistical Analysis. Data are presented as mean \pm standard deviation. The data normality was analyzed by the Kolmogorov-Smirnov test. Two-sided Student's $t$-test and one-way analysis of variance followed by Fisher's least significant difference test were performed with the SPSS 20.0 software. The differences between means were considered significant when $P<0.05$.

\section{Results}

3.1. TNF- $\alpha$ and IL-6 Were Upregulated in the AS Lesions and the ox-LDL-Stimulated Macrophages. Firstly, we examined the expression levels of CD68, TNF- $\alpha$, and IL- 6 in the lesion area from the patients with AS by IHC staining. The protein expression of CD68, TNF- $\alpha$, and IL- 6 was significantly upregulated in the lesion area from the patients with AS compared to the control group (Figures 1(a) and 1(b)). We further treated the macrophages with ox-LDL for $24 \mathrm{~h}$, and the differentiation of macrophages into foam cells was observed by oil red $\mathrm{O}$ staining (Figure 1(c)). In addition, the fluorescence microscope showed that the macrophages could directly take in Dil-oxLDL, which was red fluorescence-labeled (Figure 1(d)). The ELISA results showed that inflammatory cytokines were also significantly elevated in the macrophages induced by ox-LDL 

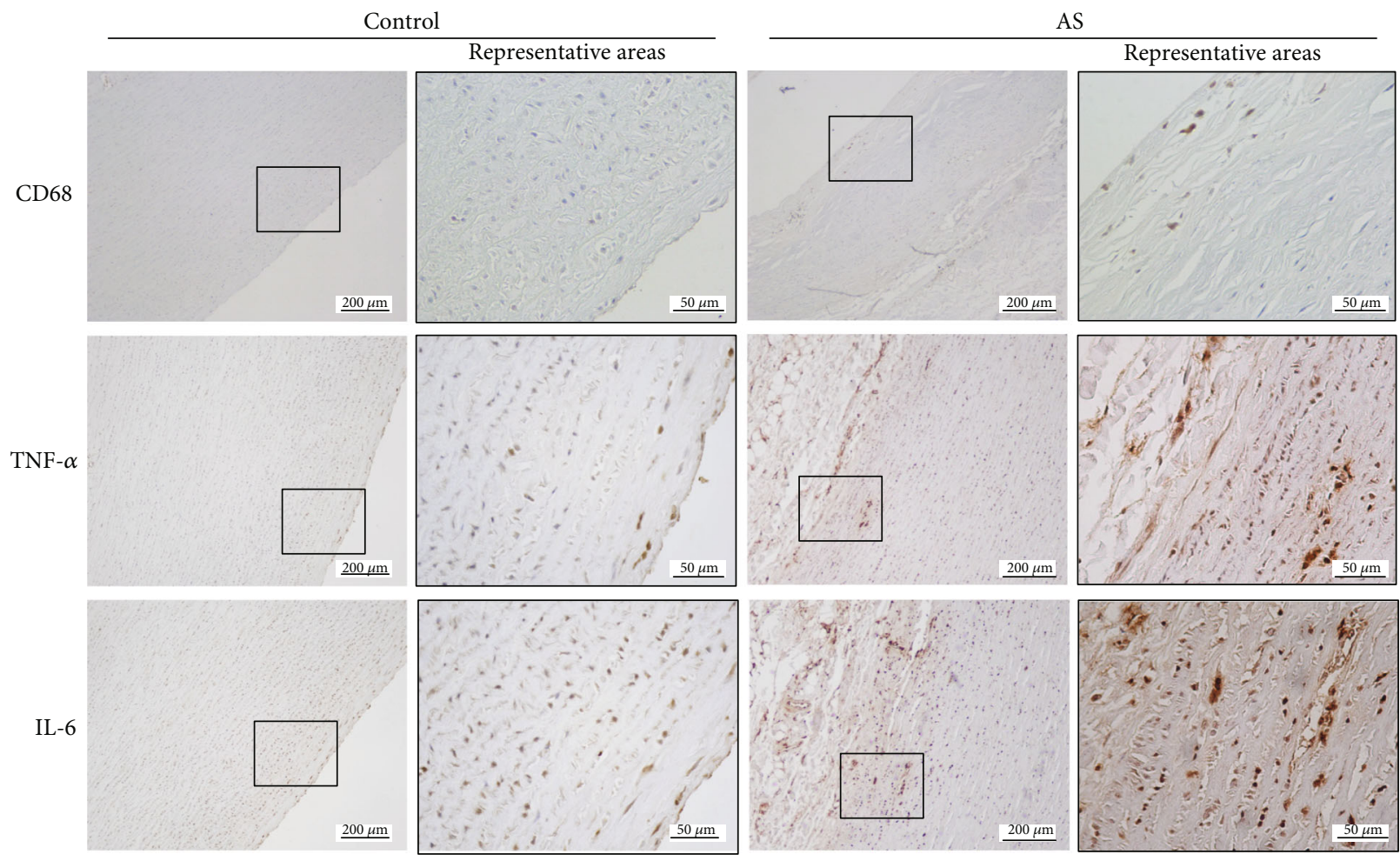

(a)

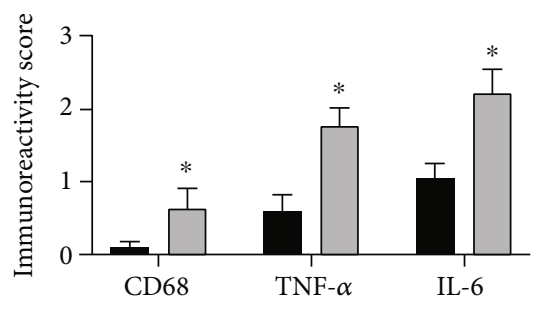

Control

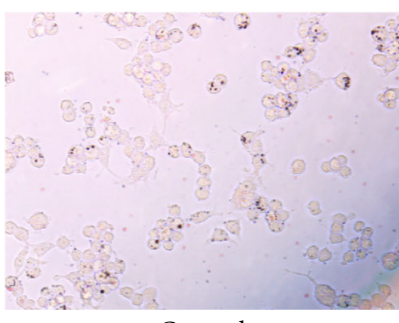

Control

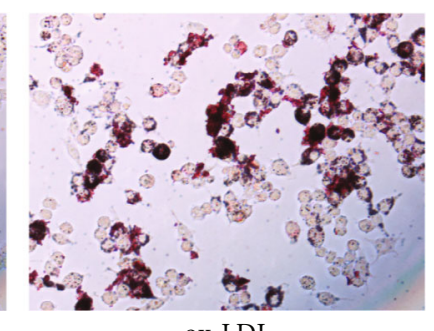

ox-LDL

(b)

(c)

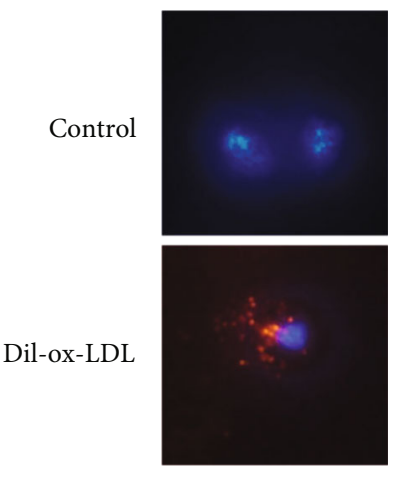

(d)

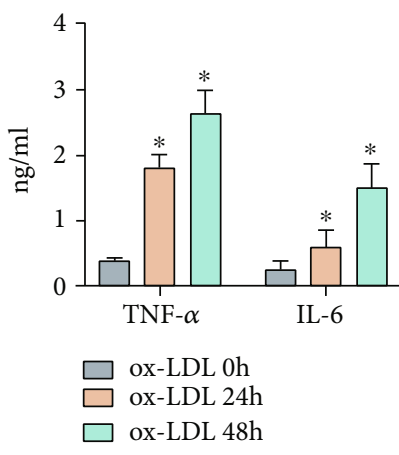

(e)

FIgure 1: Continued. 


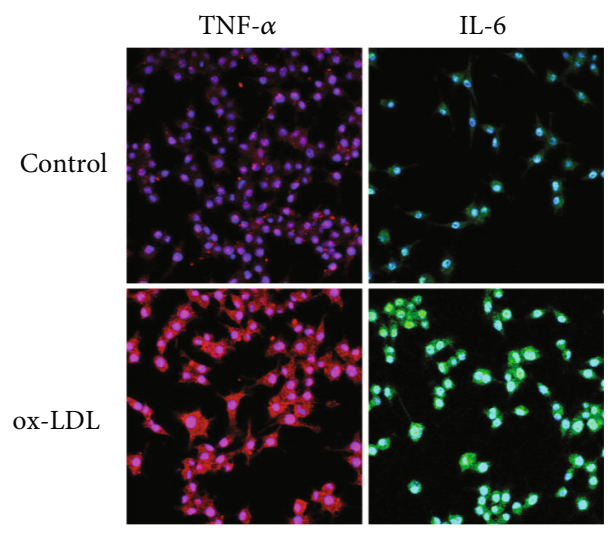

(f)

FIGURE 1: TNF- $\alpha$ and IL-6 were upregulated in the atherosclerotic aorta and the ox-LDL-stimulated macrophages. (a) The protein expression of CD68, TNF- $\alpha$, and IL- 6 in the normal aorta and atherosclerotic aorta was determined by immunofluorescent staining. (b) The immunostaining scores in the normal aorta and atherosclerotic aorta were determined. (c) The uptake of ox-LDL by macrophages was assessed by oil red $\mathrm{O}$ staining. (d) The uptake of Dil-ox-LDL by macrophages was assessed by immunofluorescent staining. (e) The TNF- $\alpha$ and IL- 6 cytokines secreted by macrophages after being treated with ox-LDL for 0,24 , and $48 \mathrm{~h}$, respectively, were determined by ELISA. (f) The TNF- $\alpha$ and IL- 6 protein expression levels in the macrophages after being treated with ox-LDL for $24 \mathrm{~h}$ were determined by immunofluorescent staining. $N=3$; significant differences between treatment groups were shown as ${ }^{*} P<0.05$.

for $24 \mathrm{~h}$ and $48 \mathrm{~h}$ (Figure $1(\mathrm{e})$ ). In addition, the mRNA levels of TNF- $\alpha$ and IL- 6 were upregulated and induced by ox-LDL (Fig. S1A). Consistently, the fluorescent signaling intensities of these two inflammatory proteins significantly increased in macrophages because of the treatment of ox-LDL (Figure 1(f)).

3.2. ox-LDL Increased the Oxidative Stress and Mitochondrial Injury via Modulating NRF2. Here, ox-LDL treatment significantly enhanced the ROS production in the macrophages and also raised the number of ROS-positive macrophages (Figures 2(a) and 2(b) and Fig. S1B). The Western blot results showed that ox-LDL stimulation significantly raised the protein level of NRF2 in the nucleus of macrophages but decreased NRF2 and KEAP1 protein levels in the cytoplasm of the macrophages (Figures 2(c) and 2(d) and Fig. S1CS1D). Moreover, the MitoTracker staining showed that oxLDL stimulation significantly induced the mitochondrial injury of the macrophages (Figure 2(e)). Besides, the oxLDL stimulation for 4 and $8 \mathrm{~h}$ both increased the protein levels of DRP1 and FIS1 in the macrophages (Figure 2(f) and Fig. S1E).

3.3. ZBTB20 Was Identified as the Hub Gene Associated with Oxidative Stress and Inflammation in ox-LDL-Stimulated Macrophages. The DEGs between the treatment group and the control group from the GSE54666 dataset were illustrated in the volcano plot and heat map, respectively (Figures 3(a) and 3(b)). A total of 642 DEGs were identified, including 357 upregulated DEGs and 285 downregulated DEGs. The DEGs were then subjected to the PPI network construction by using the STRING database, and a total of 538 nodes and 2037 edges were identified in the PPI network. The top 15 significant GO terms and KEGG pathways from the GO categories and KEGG database were shown in Fig. S2A, and the four most significant KEGG pathways analyzed with GSEA were shown in Fig. S2B. Furthermore, the hub genes were extracted using the MCODE application in the Cytoscape software. Eighty-six hub genes were identified, and the ZBTB20 gene had a high score among them (Figure 3(c)). Besides, the hub gene network was obtained according to the core gene scores by the cytoHubba plug-in (Figure 3(d)). The functional clustering of the hub genes showed that ZBTB20 was associated with oxidative stress, inflammation, and cytokines (Figure 3(e)).

3.4. ZBTB20 Promoted the Inflammatory Responses of the oxLDL-Stimulated Macrophages. The expression of ZBTB20 as illustrated by the IHC staining was significantly upregulated in the lesion area from the patients with AS compared to the control group (Figure 4(a)). Western blot as well as qRT-PCR showed that ox-LDL time-dependently increased the expression of ZBTB20 in the macrophages not only in the mRNA level but also in the protein level (Figures 4(b) and 4(c) and Fig. S3A). The Western blot result of ZBTB20-siRNA knockdown efficiency was shown in Fig. S3B. The ELISA results showed that the knockdown of ZBTB20 significantly reduced the inflammatory cytokine levels secreted by ox-LDL-induced macrophages (Figure 4(d)). Consistently, the ZBTB20 knockdown significantly reduced the fluorescent signaling intensities of TNF- $\alpha$ and IL- 6 proteins in ox-LDL-induced macrophages (Figure 4(e)).

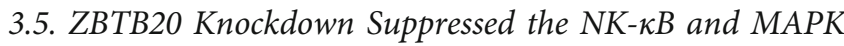
Signaling Activities in the ox-LDL-Stimulated Macrophages. Here, the effects of the ZBTB20 knockdown on the ox-LDL uptake of macrophages were determined by fluorescent staining and flow cytometry. As shown in Figures 5(a) and 5(b) and Fig. S3C, the ZBTB20 knockdown had no significant effects on the Dil-ox-LDL-positive macrophage ratios as determined by flow cytometry. Consistently, there was no significant difference in the fluorescent signaling intensity of Dil-ox-LDL in the macrophages between the NC-siRNA 


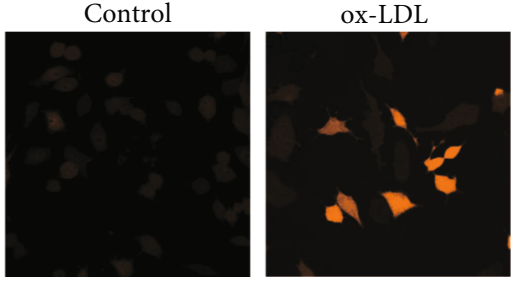

(a)

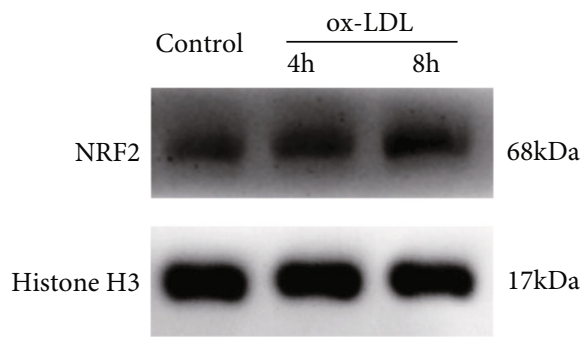

(c)
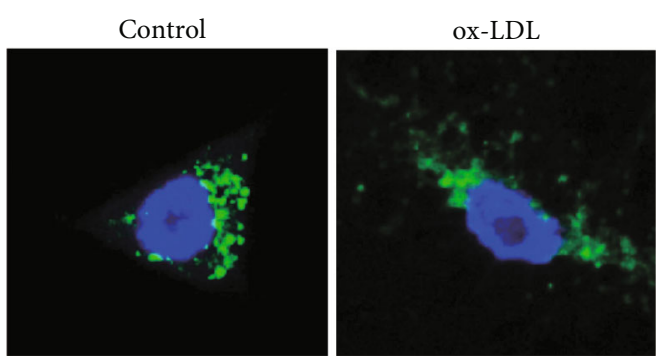

(e)
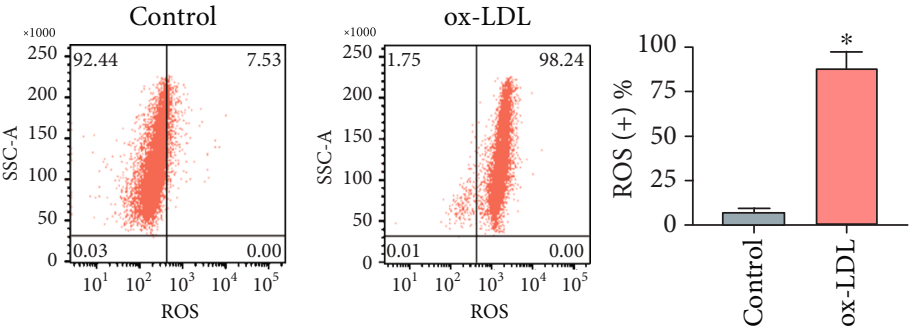

(b)

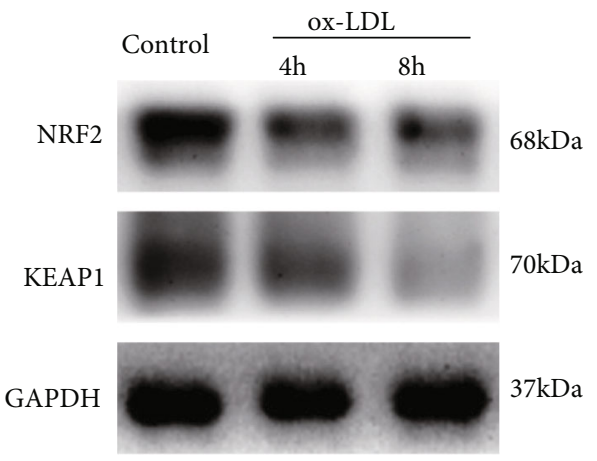

(d)

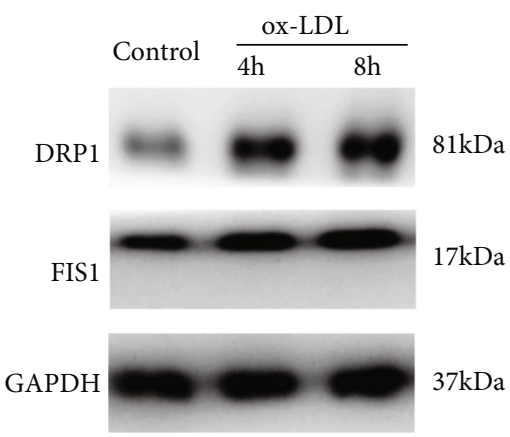

(f)

FIGURE 2: ox-LDL increased the oxidative stress and mitochondrial injury via modulating NRF2. (a) The ROS level of the PBS- or ox-LDLtreated macrophages was determined by immunofluorescent staining. (b) The ROS-positive rates of the PBS- or ox-LDL-treated macrophages with different treatments were determined by flow cytometry. (c) The protein level of NRF2 in the nucleus of the PBS- or ox-LDL-treated macrophages was assessed by Western blot assay. (d) The protein level of NRF2 and KEAP1 in the cytoplasm of the PBS- or ox-LDLtreated macrophages was assessed by Western blot assay. (e) The mitochondrial injury of the PBS- or ox-LDL-treated (6h) macrophages was assessed by MitoTracker staining. (f) The protein level of DRP1 and FIS1 in the PBS- or ox-LDL-treated (6h) macrophages with respective treatments was assessed by Western blot assay. $N=3$; significant differences between treatment groups were shown as ${ }^{*} P<0.05$.

and ZBTB20-siRNA groups as determined by flow cytometry (Figure 5(c)).

The effects of the ZBTB20 knockdown on the protein levels of the NK- $\kappa \mathrm{B}$-related mediators in the macrophages were determined by the Western blot assay. Interestingly, the ZBTB20 knockdown significantly reduced the protein level of p-p65 but increased that of $\mathrm{I} \kappa \mathrm{B} \alpha$ in the ox-LDLstimulated macrophages when compared to the PBS-treated macrophages (Figure 5(d) and Fig. S3D). Consistently, the fluorescent staining showed that the ZBTB20 knockdown suppressed the nucleus translocation of p65 in the ox-LDLstimulated macrophages (Figure 5(e)). In addition, the MAPK-related mediators were also examined, and as shown in Figure 5(f) and Fig. S3E, the ZBTB20 knockdown signifi- cantly downregulated the phosphorylation levels of JNK, ERK, and p38 induced by ox-LDL in macrophages.

3.6. ZBTB20 Knockdown Suppressed M1 Polarization, Increased M2 Polarization, and Inhibited the Phosphorylation and Nucleus Translocation of IRF3. Here, the effects of the ZBTB20 knockdown on M1/M2 polarization of macrophages were determined by flow cytometry, and as presented in Figure 6(a) and Fig. S4A-S4B, the percentage of macrophages with $\mathrm{M} 1$ polarization significantly decreased, and the percentage of macrophages with M2 polarization increased in the ZBTB20-siRNA group compared to the NC-siRNA group. The qRT-PCR results showed that the ZBTB20 knockdown downregulated the mRNA level of iNOS, an M1 polarization 

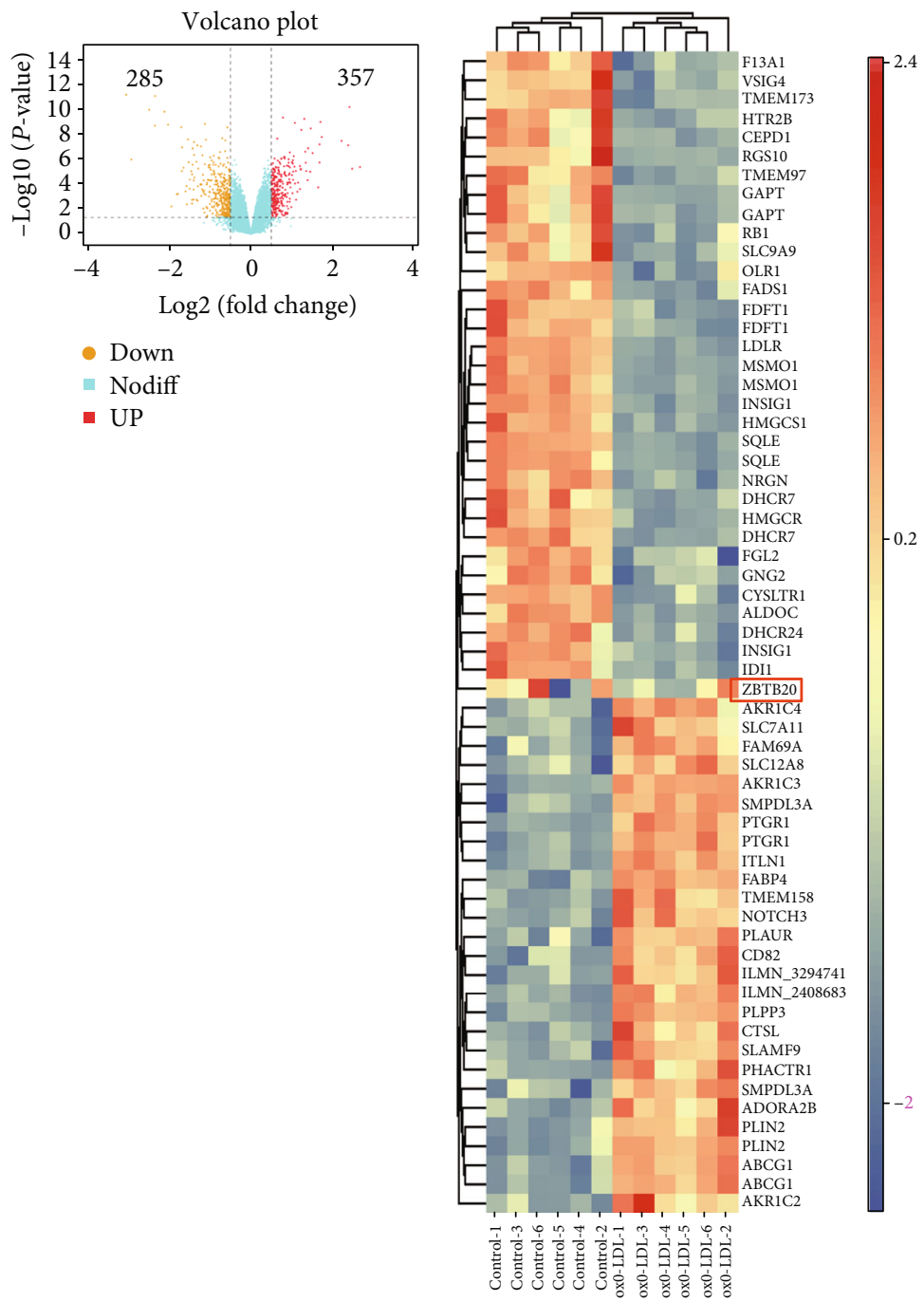

(a)

(b)

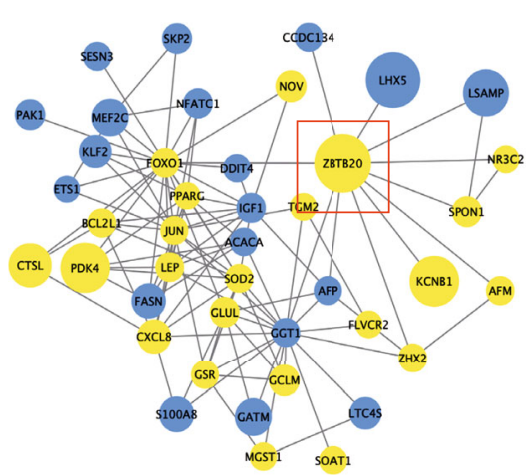

Upregulated mRNAs

- Downregulated mRNAs

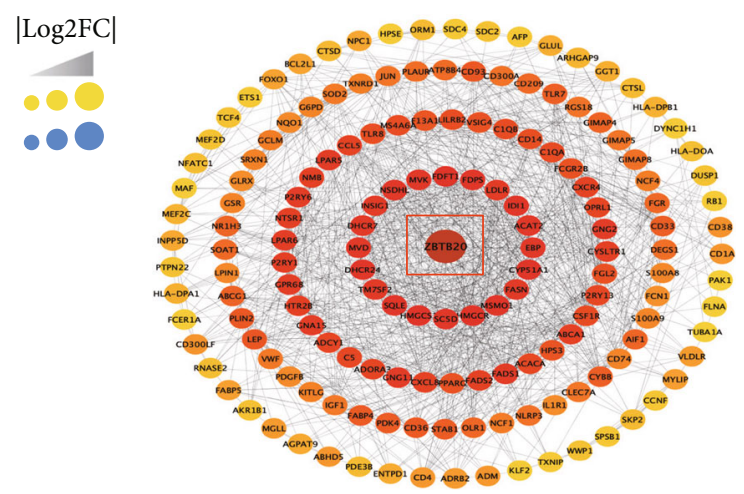

(d)

Figure 3: Continued. 


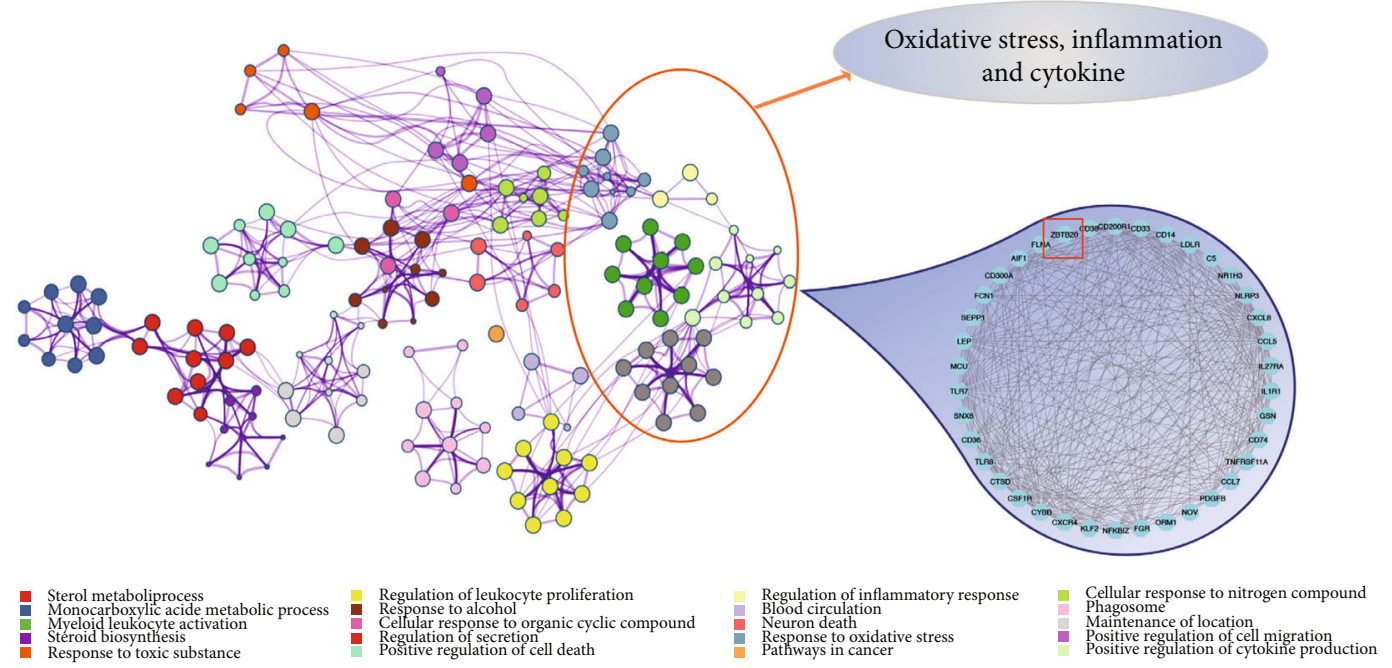

(e)

Figure 3: ZBTB20 was identified as the hub gene associated with oxidative stress, inflammation, and cytokines in the ox-LDL-stimulated macrophages. (a) Volcano plots and (b) heat map illustration of the differentially expressed genes between control macrophages and oxLDL-stimulated macrophages. (c) The hub genes from the PPI network were further identified using the MCODE app from Cytoscape software. (d) The cytoHubba plug-in from Cytoscape software was used, and the hub gene network was obtained according to the core gene scores. (e) The functional clustering of genes associated with oxidative stress, inflammation, and cytokines was performed using the Metascape database.

marker, but upregulated the mRNA level of COX-2, an M2 polarization marker, in the ox-LDL-stimulated macrophages (Figure 6(b)). Interestingly, the Western blot results showed that the ZBTB20 knockdown significantly suppressed the phosphorylation of the IRF3 in the ox-LDL-stimulated macrophages, but not in the PBS-treated macrophages (Figure 6(c) and Fig. S4C). In addition, the fluorescent staining results showed that the ZBTB20 knockdown repressed the nucleus translation of IRF3 in the ox-LDL-stimulated macrophages (Figure 6(d)). ELISA showed that the ZBTB20 knockdown reduced the cytokine levels of IFN- $\beta$ in the ox-LDLstimulated macrophages (Figure 6(e)).

\subsection{ZBTB20 Regulated Oxidative Stress and Mitochondrial} Fission in ox-LDL-Stimulated Macrophages via Modulating NRF2. The ROS production in the macrophages with respective treatments was analyzed using fluorescent staining and flow cytometry. The fluorescent staining results showed that the treatment of ox-LDL significantly increased the ROS level in the macrophages, which was significantly attenuated by the ZBTB20 knockdown (Figure 7(a)). Consistently, the ZBTB20 knockdown attenuated the ox-LDL-induced increase of ROSpositive macrophages, and more importantly, the effects of the ZBTB20 knockdown on the number of ROS-positive macrophages were antagonized by the treatment of ML385, a novel NRF2 inhibitor (Figures 7(b) and 7(c) and Fig. S4D). The fluorescent staining results showed that the knockdown of ZBTB20 enhanced the nucleus translocation of NRF2 induced by ox-LDL, which was also attenuated by ML385 treatment (Figure 7(d)). Consistently, the ZBTB20 knockdown increased the nuclear expression level of NRF2 protein in ox-LDLstimulated macrophages as determined by the Western blot assay (Figure 7(e) and Fig. S4E). Furthermore, MitoTracker staining showed that the ZBTB20 knockdown prevented the mitochondrial injury in the macrophages (Figure $7(f)$ ), and the increased protein expression levels of DRP1 and FIS1 induced by ox-LDL were significantly attenuated by the ZBTB20 knockdown as well (Figure 7(g) and Fig. S4F-S4G).

3.8. Knockdown of ZBTB20 Attenuates the Development of AS in $A p o E^{-/-}$Mice. The effects of the ZBTB20 knockdown on the progression of AS were evaluated in $\mathrm{ApoE}^{-/-}$mice, which were fed with a high-fat diet for 12 weeks. As shown in Figure 8(a), the tail vein injection of the adenovirus expressing sh-ZBTB20 significantly reduced the aorta lesion in the $\mathrm{ApoE}^{-/-}$mice when compared to animals treated with control adenovirus. The H\&E staining showed consistent results (Figure 8(b)). Furthermore, the foam cells in the aorta were stained by the oil red, and the lesion area significantly decreased in the sh-ZBTB20 group (Figure 8(c)). Besides, we performed Masson staining for collagen content, IHC staining for smooth muscle cell (SMC) content ( $\alpha$-SMA $(\alpha$-smooth muscle actin)), and macrophage accumulation (CD68) in whole aortas from sh-NC-treated $\mathrm{ApoE}^{-/-}$mice and shZBTB20-treated $\mathrm{ApoE}^{-/-}$mice (Figure $8(\mathrm{~d})$ ), and the results showed that the knockdown of ZBTB20 attenuated the macrophage accumulation in the progression of AS but had no effects on collagen or SMC content.

\section{Discussion}

AS progression is closely related to proinflammatory and proatherogenic mediators, which can promote plaque formation and stenosis progression [39]. In the initiation of AS, high levels of ox-LDL can recruit monocytes, promoting the 


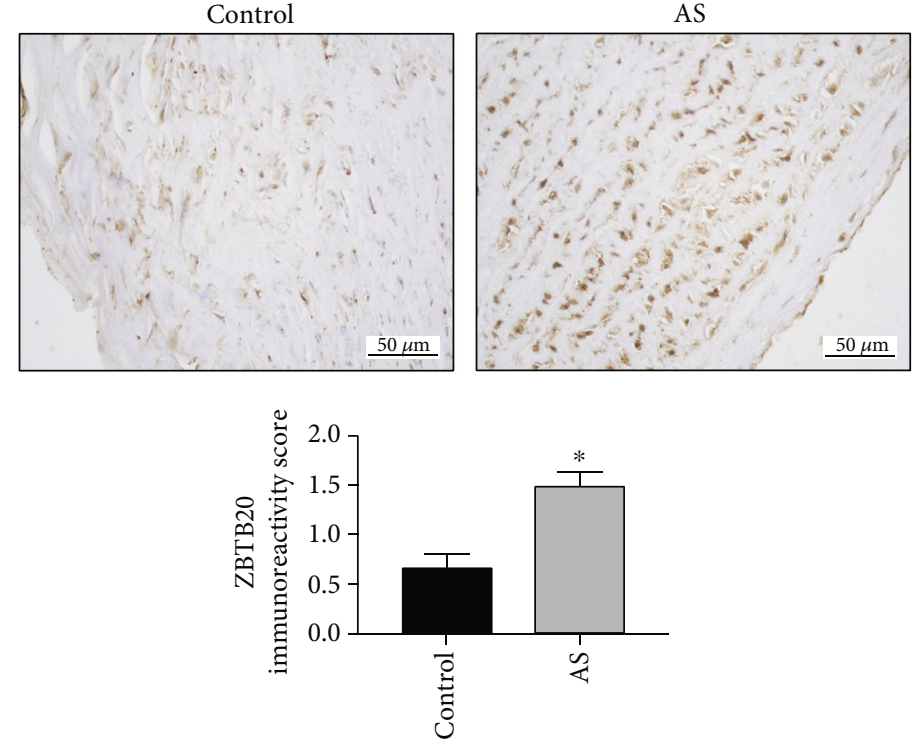

(a)

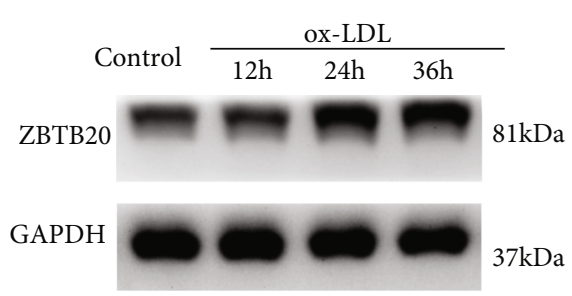

(c)

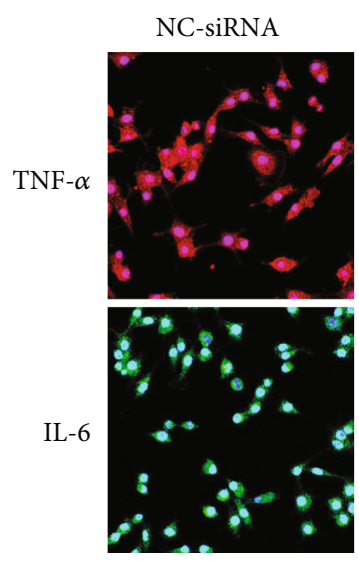

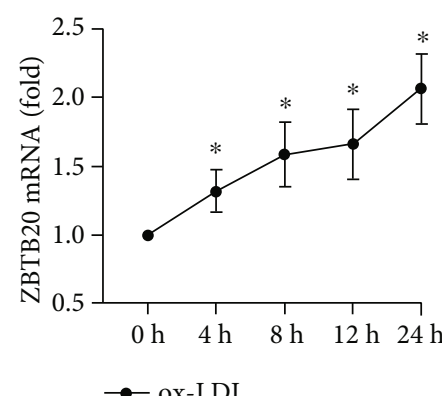

(b)
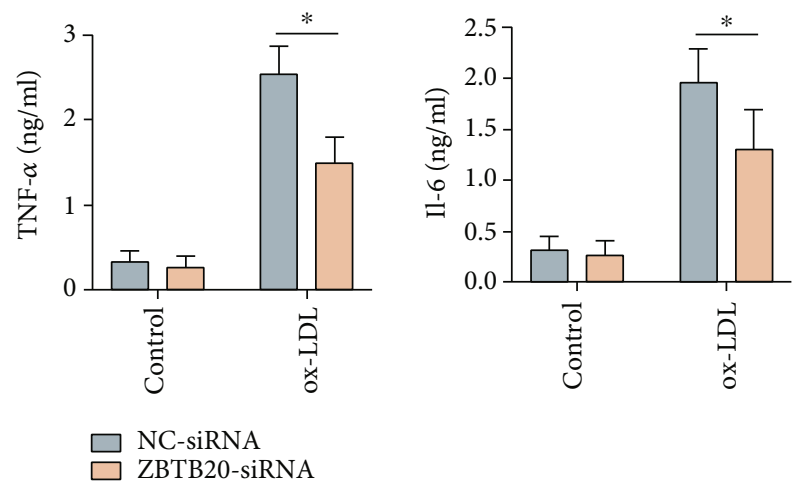

(d)

ZBTB20-siRNA

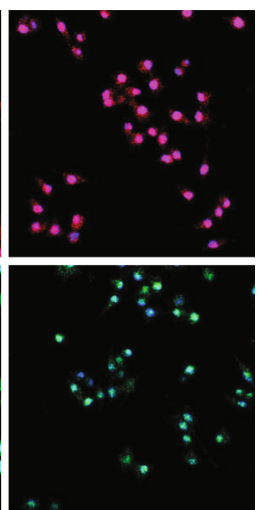

(e)

FIGURE 4: ZBTB20 promotes the inflammatory responses of the ox-LDL-stimulated macrophages. (a) The protein expression of ZBTB20 in the normal aorta and atherosclerotic aorta was determined by immunofluorescent staining. The (b) mRNA and (c) protein expression levels of ZBTB20 in the macrophages after being treated with ox-LDL for 0, 4, 8, or $24 \mathrm{~h}$ were determined by qRT-PCR and Western blot assays, respectively. (d) The TNF- $\alpha$ and IL-6 protein expression levels in the PBS- or ox-LDL-treated macrophages after being transfected with scrambled siRNA (NC) or si-ZBTB20 were determined by ELISA. (e) The TNF- $\alpha$ and IL-6 protein expression levels in the ox-LDL-treated macrophages after being transfected with scrambled siRNA (NC) or si-ZBTB20 were evaluated by immunofluorescent staining. $N=3$; significant differences between treatment groups were shown as ${ }^{*} P<0.05$. 

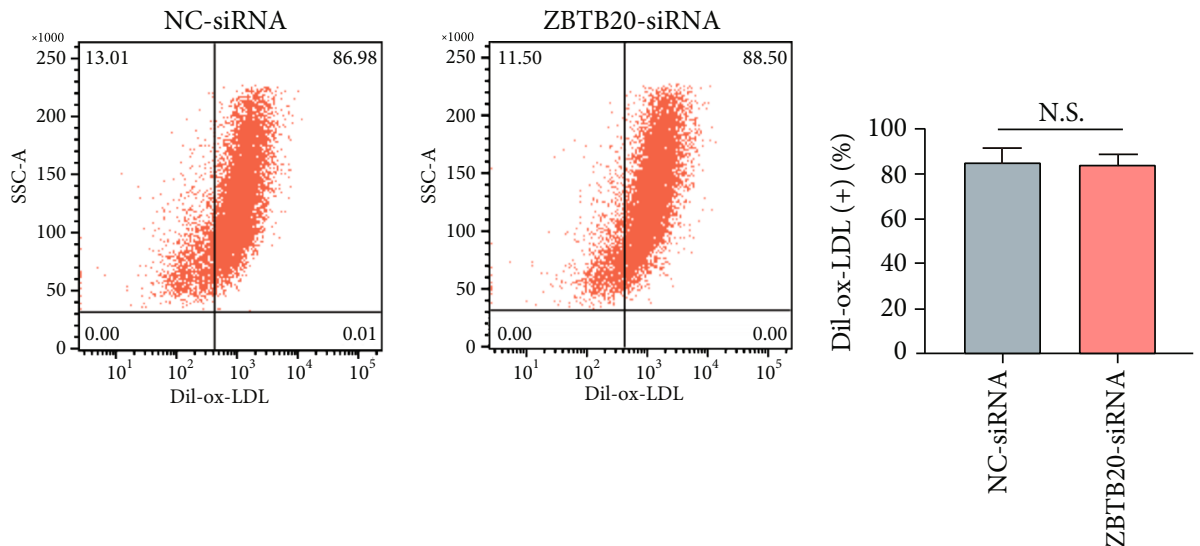

(a)

(b)
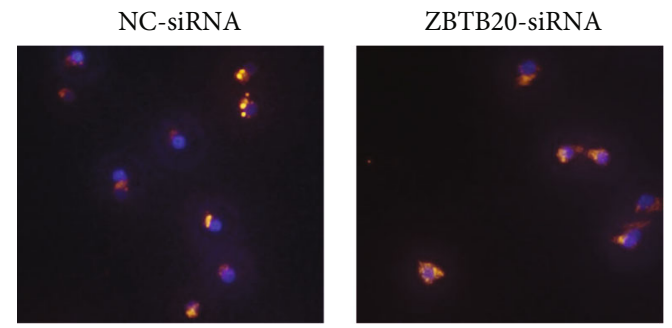

(c)
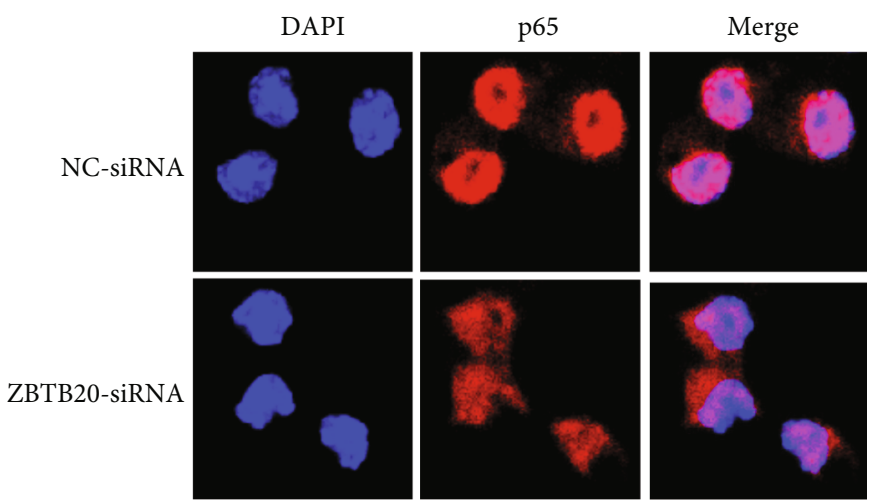

(e)

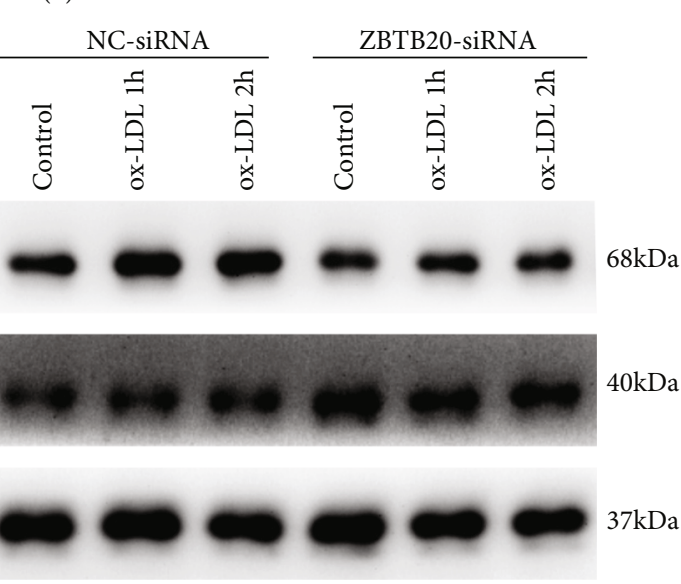

(d)
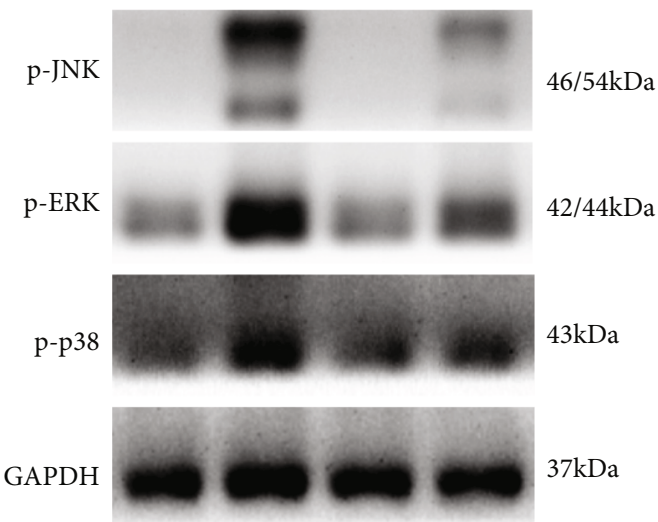

(f)

FIGURE 5: ZBTB20 knockdown suppresses the NF- $\kappa$ B and MAPK signaling activities in the ox-LDL-stimulated macrophages. (a, b) The oxLDL uptake of macrophages after being transfected with scrambled siRNA (NC) or si-ZBTB20 was determined by flow cytometry. (c) The oxLDL uptake of macrophages after being transfected with scrambled siRNA (NC) or si-ZBTB20 was determined by immunofluorescent staining. (d) The protein expression levels of the NF- $\kappa B$-related mediators in the PBS- or ox-LDL-treated macrophages after being transfected with scrambled siRNA (NC) or si-ZBTB20 were determined by Western blot assay. (e) The protein expression level of p65 in ox-LDL-treated $(1 \mathrm{~h})$ macrophages after being transfected with scrambled siRNA (NC) or si-ZBTB20 was determined by immunofluorescent staining. (f) The protein expression levels of the MAPK-related mediators in the PBS- or ox-LDL-treated (1 h) macrophages after being transfected with scrambled siRNA (NC) or si-ZBTB20 were determined by Western blot assay. $N=3$. 

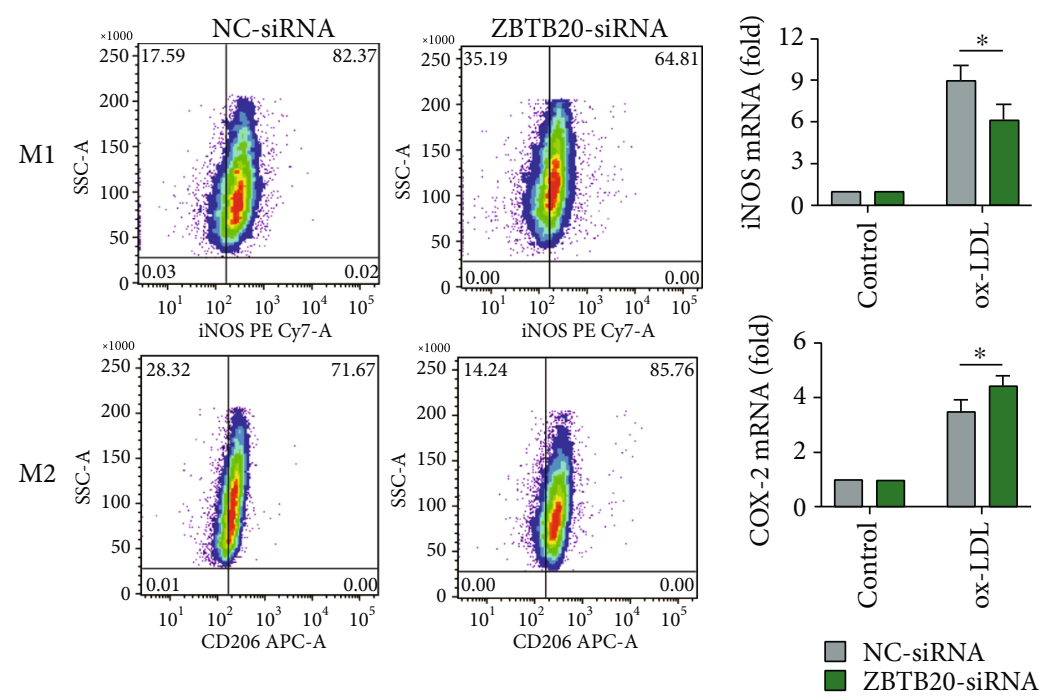

(a)

(b)
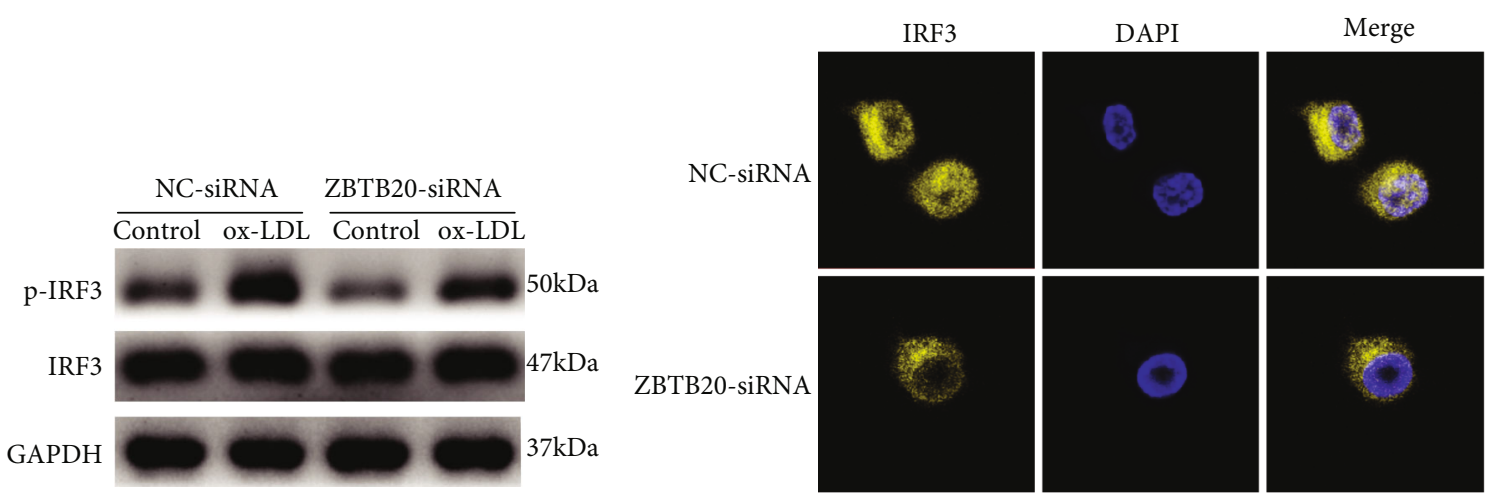

(c)

(d)

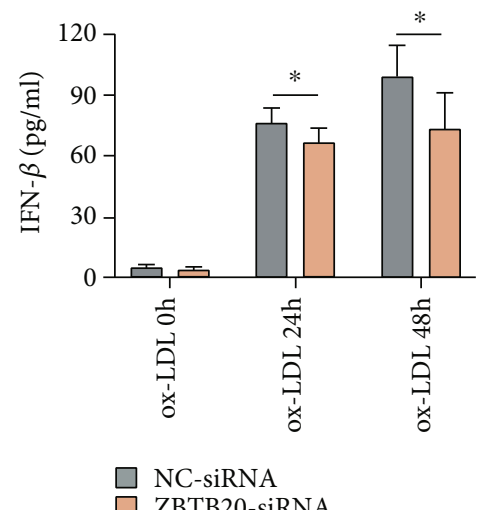

(e)

Figure 6: ZBTB20 knockdown suppresses the M1 polarization, increases the M2 polarization, and inhibits the phosphorylation and nucleus translocation of IRF3 in the ox-LDL-stimulated macrophages. (a) The M1 and M2 polarization of the ox-LDL-treated (24h) macrophages after being transfected with scrambled siRNA (NC) or si-ZBTB20 was determined by flow cytometry. (b) The mRNA expression level of the iNOS in the PBS- or ox-LDL-treated $(24 \mathrm{~h})$ macrophages after being transfected with scrambled siRNA (NC) or si-ZBTB20 was determined by qRT-PCR. (c) The phosphorylation level of the IRF3 in the PBS- or ox-LDL-treated (8h) macrophages after being transfected with scrambled siRNA (NC) or si-ZBTB20 was determined by Western blot assay. (d) The nucleus translocation of IRF3 in the ox-LDL-treated $(8 \mathrm{~h})$ macrophages after being transfected with scrambled siRNA (NC) or si-ZBTB20 was determined by immunofluorescent staining. (e) The cytokine level of IFN- $\beta$ in the ox-LDL-treated macrophages after being transfected with scrambled siRNA (NC) or si-ZBTB20 was determined by ELISA. $N=3$; significant differences between treatment groups were shown as ${ }^{*} P<0.05$. 


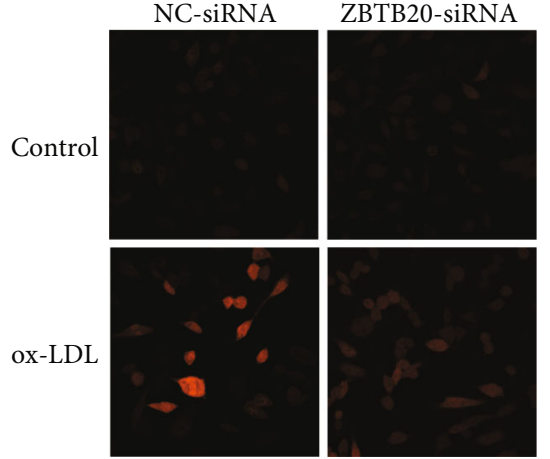

(a)

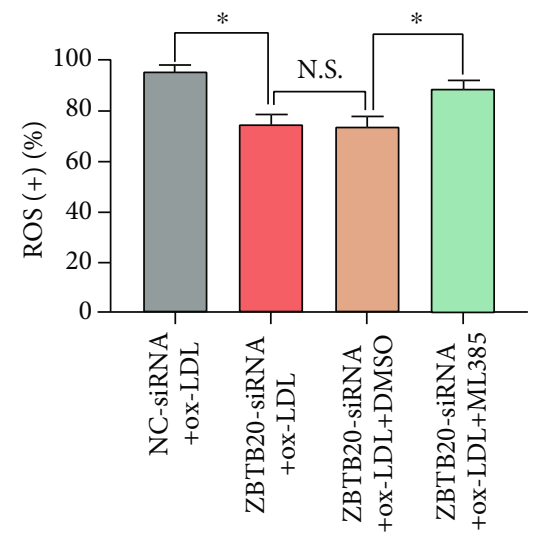

(c)
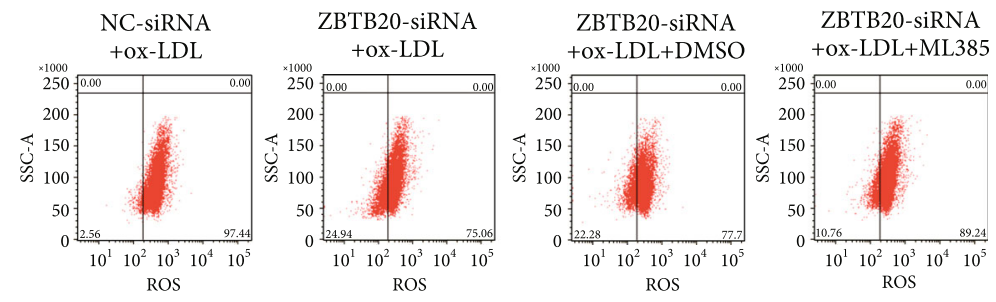

(b)

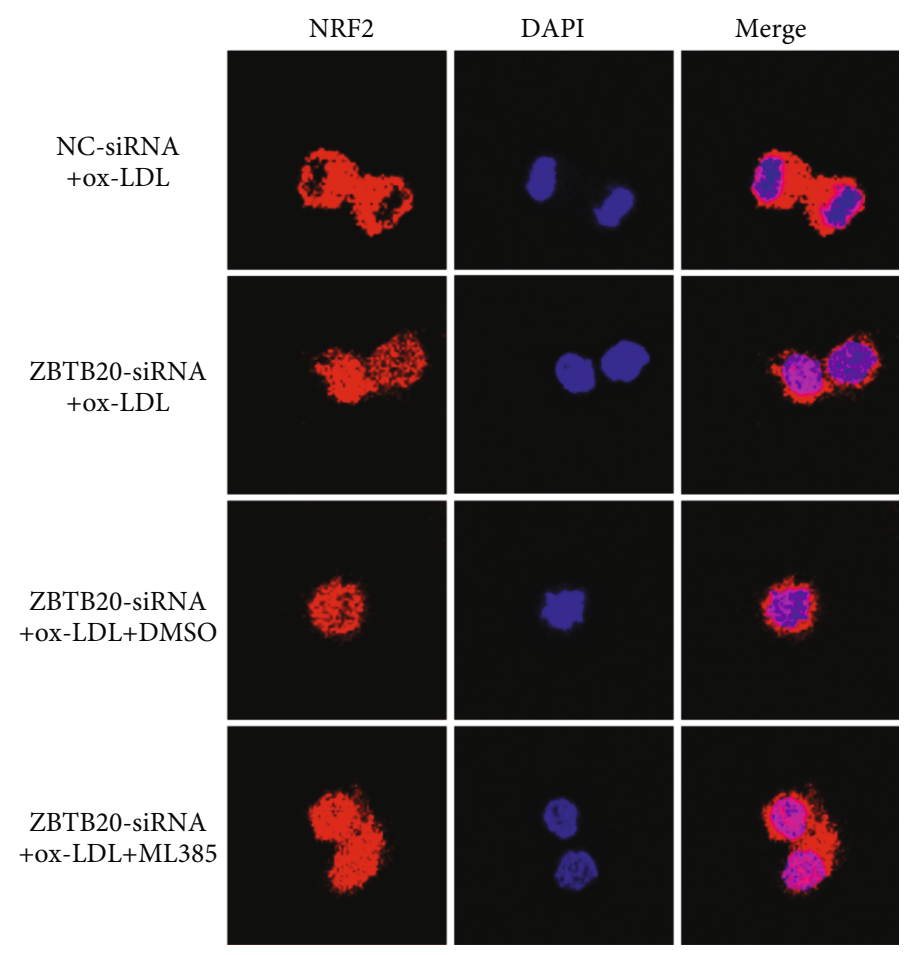

(d)
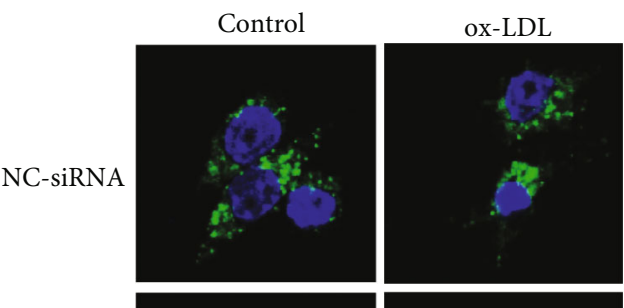

$\frac{\text { NC-siRNA }}{\text { Control ox-LDL }} \quad$ ZBTB20-siRNA

NRF2 $68 \mathrm{kDa}$ ZBTB20-siRNA $17 \mathrm{kDa}$ Histone $\mathrm{H} 3$

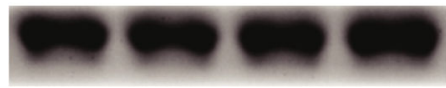

(e)

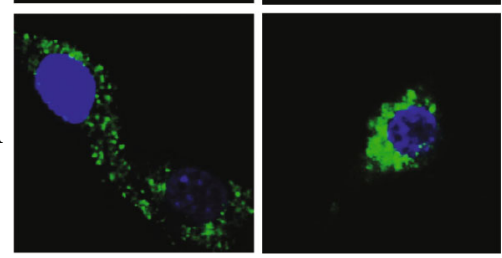

(f)

Figure 7: Continued. 


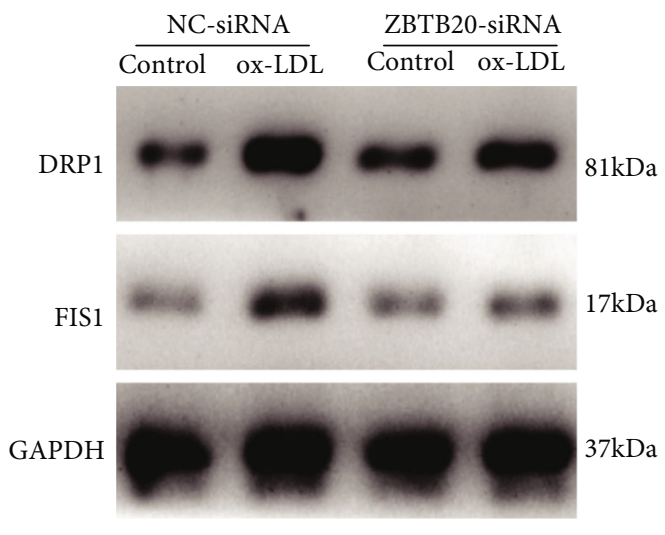

$(\mathrm{g})$

FIGURE 7: ZBTB20 positively regulates oxidative stress and mitochondrial fission in ox-LDL-stimulated macrophages via modulating NRF2. (a) The ROS level in the PBS- or ox-LDL-treated macrophages after being transfected with scrambled siRNA (NC) or si-ZBTB20 was determined by immunofluorescent staining. (b, c) The ROS-positive rates of the macrophages with different treatments were determined by flow cytometry. (d) The nucleus translation of NRF2 in the ox-LDL-stimulated macrophages with respective treatments was determined by immunofluorescent staining. (e) The protein level of NRF2 in the nucleus of the macrophages with respective treatments was assessed by Western blot assay. (f) The mitochondrial injury of the macrophages with respective treatments was assessed by MitoTracker staining. (g) The protein level of DRP1 and FIS1 in the macrophages with respective treatments was assessed by Western blot assay. $N=3$; significant differences between treatment groups were shown as ${ }^{*} P<0.05$.

adhesion molecule expression on the endothelium and the subsequent adhesion of the monocytes to the intima [40]. Here, our microarray analysis revealed that ZBTB20 was identified as the hub gene in the ox-LDL-stimulated macrophages. The validation studies showed that ZBTB20 was upregulated in the human AS lesions and ox-LDL-stimulated macrophages. The loss-of-function studies showed that the ZBTB20 knockdown suppressed the proinflammatory cytokine levels, decreased the M1 polarization, and reduced the oxidative stress and mitochondrial fission in the ox-LDL-stimulated macrophages. The mechanistic studies showed that the ZBTB20 knockdown not only suppressed the NK- $\kappa \mathrm{B}$ and MAPK signaling activities but also inhibited the nucleus translocation of NRF2 in the ox-LDL-stimulated macrophages. Our in vivo data showed that the ZBTB20 knockdown attenuated the development of $\mathrm{AS}$ in $\mathrm{ApoE}^{-/-}$mice.

The present study showed the increased expression levels of proinflammatory factors in the human AS lesions and oxLDL-stimulated macrophages, which was consistent with previous studies $[41,42]$. These results indicated the increased inflammatory response in the macrophages during AS. The upregulation of ZBTB20 in human AS lesions and ox-LDLstimulated macrophages suggested that ZBTB20 may promote the initiation and progression of AS. Thus, we performed the loss-of-function studies by silencing the ZBTB20 gene. In this study, the knockdown of ZBTB20 suppressed proinflammatory protein levels in ox-LDL-induced macrophages, which was consistent with studies by Qiu et al., showing that ZBTB20 silencing suppressed the inflammatory responses in titanium particle-stimulated or lipopolysaccharide- (LPS-) stimulated macrophages [18]. In addition, the ZBTB20 knockout was reported to decrease the serum levels of IL- 6 and TNF- $\alpha$ in LPS-treated mice [20]. AS is an inflammatory disease, and $\mathrm{NF}-\kappa \mathrm{B}$ functions as a major transcription factor in inflamma- tory and immune responses $[43,44]$. Thus, we further examined the roles of the ZBTB20 knockdown on NF- $\kappa \mathrm{B}$ activities, and we found that the ox-LDL stimulation increased the activities of macrophage NF- $\kappa \mathrm{B}$ signaling, which was consistent with findings from previous studies $[45,46]$. ZBTB20 was also found to promote the activity of NF- $\kappa \mathrm{B}$ in the gastric cancer cells and human dental pulp stem cells [47, 48]. Besides, the present study showed that ox-LDL enhanced the activity of MAPK, another inflammatory signaling, in macrophages, which was consistent with previous reports from Taketa et al. [49]. Here, our further results revealed that the ZBTB20 knockdown reduced MAPK activity in ox-LDLstimulated macrophages. However, studies from Liu et al. showed that ZBTB20 had no effects on Toll-like receptortriggered activation of MAPK [20]. The inconsistent effects of ZBTB20 on the activity of MAPK in macrophages among different studies might be attributed to the different stimuli used. Collectively, in this study, the inhibitory effects of the ZBTB20 knockdown on the inflammatory responses in the macrophages may be related to the impaired activity of NF$\kappa \mathrm{B}$ and MAPK.

Macrophages can be divided into M1 or M2 type, depending on the polarization state, all of which were derived from monocytes [50]. Recent studies showed that M2 macrophages could clear dying cells and debris and secrete antiinflammatory factors, which can attenuate the formation of AS plaques [51]. Our results showed that the ZBTB20 knockdown increased the M2 macrophage polarization but decreased the M1 macrophage polarization, implying that the ZBTB20 knockdown may attenuate AS by activating M2 macrophages. IRF3 is a key interferon-regulator factor in regulating the $\mathrm{M} 2$ polarization of macrophages, and IRF3 can cooperate with NF- $\kappa$ B to launch IFN- $\beta$ gene transcription [52]. Consistently, our data showed that the 


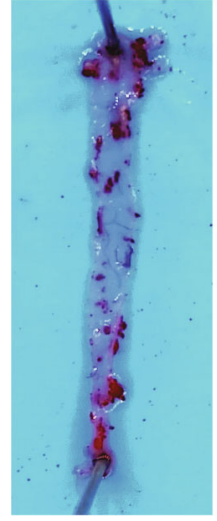

sh-NC

adenovirus

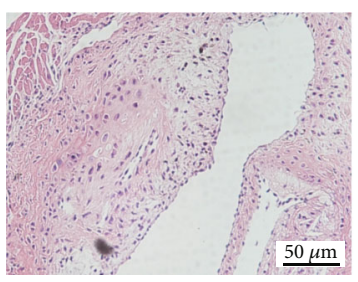

sh-NC

adenovirus

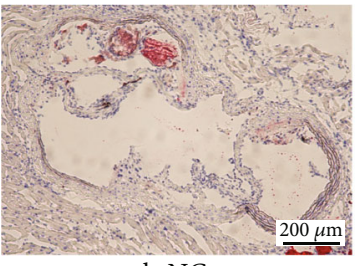

sh-NC

adenovirus

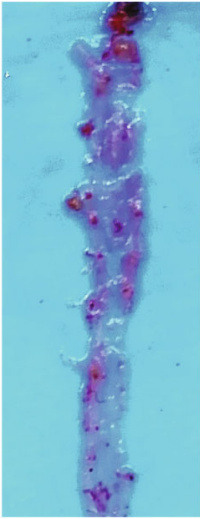

sh-ZBTB20

adenovirus

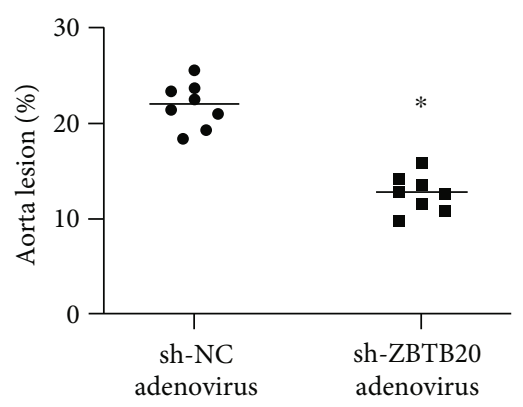

(a)

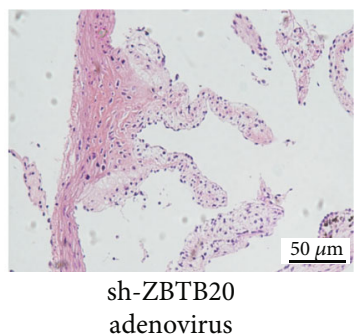

(b)

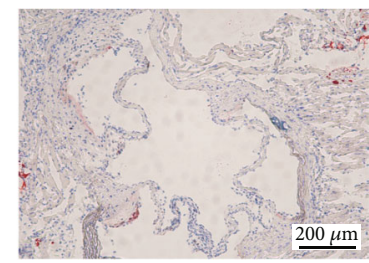

sh-ZBTB20

adenovirus
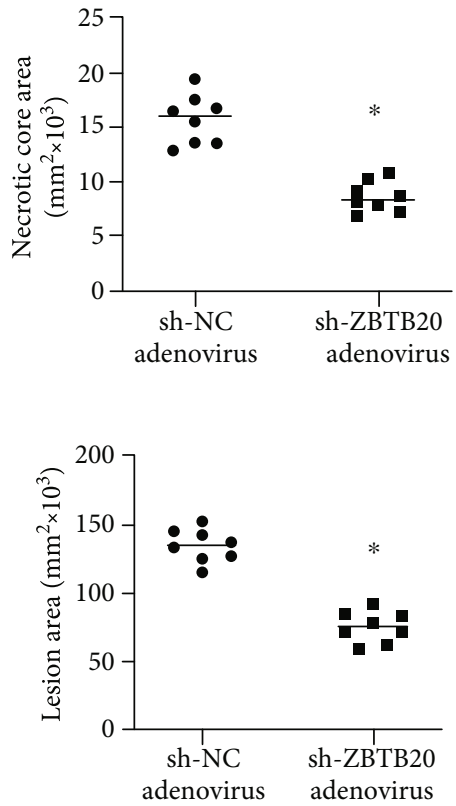

(c)
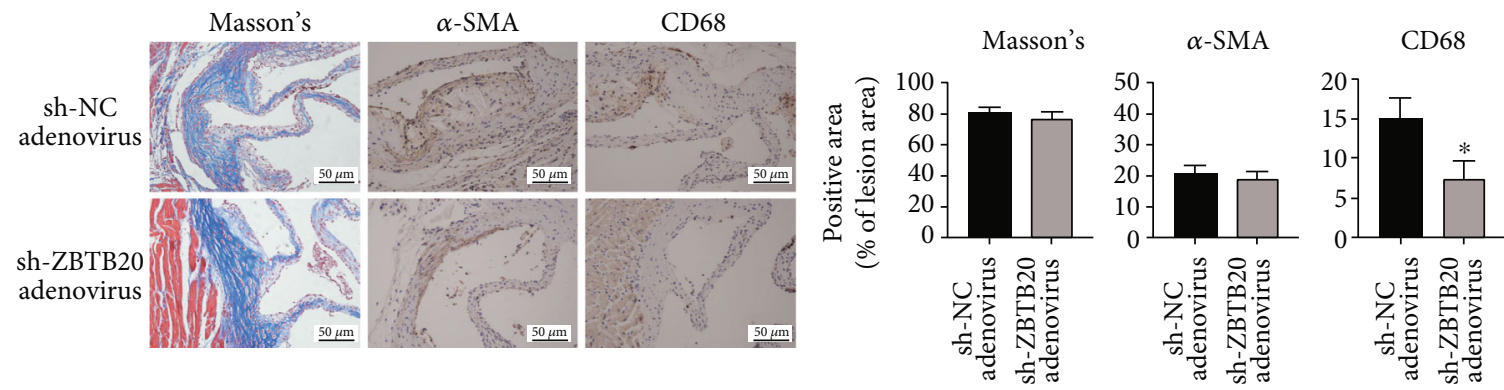

(d)

FigURE 8: Knockdown of ZBTB20 attenuates the progression of the atherosclerosis of the ApoE ${ }^{-/-}$mice. (a) Representative images of the oil red $\mathrm{O}$ staining in whole aortas from sh-NC-treated $\mathrm{ApoE}^{-/-}$mice or sh-ZBTB20-treated ApoE ${ }^{-/-}$mice fed with a high-fat diet for 16 weeks. ImageJ software was used to quantify the lesion coverage of the entire aorta (\%). (b) Representative images of H\&E staining in crosssections of the aortic root from mice in different groups. ImageJ software was used to quantify areas of the necrotic core. (c) Representative images of oil red $\mathrm{O}$ staining in cross-sections of the aortic root from mice in different groups. ImageJ software was used to assess the lesion areas. (d) Representative images of Masson staining for collagen content, IHC staining for SMC content, and macrophage accumulation (CD68) in whole aortas from sh-NC-treated ApoE ${ }^{-/-}$mice or sh-ZBTB20-treated ApoE ${ }^{-/-}$mice fed with a high-fat diet for 16 weeks. $N=8$; significant differences between treatment groups were shown as ${ }^{*} P<0.05$. 
ZBTB20 knockdown suppressed the phosphorylation and nucleus translocation of IRF3 and reduced the IFN- $\beta$ cytokine level in the ox-LDL-stimulated macrophages, suggesting that ZBTB20 regulated the phenotype switching of macrophages by targeting IRF3 and regulating IFN- $\beta$. Similarly, in the clinic, it may be possible to use macrophage polarization as a target to treat macrophage-related inflammatory diseases, such as aseptic loosening and immune rejection.

It has been widely reported that ox-LDL induced oxidative stress in macrophages [53, 54], and our results consistently elucidated that ox-LDL increased the ROS production and DRP1/FIS1 protein. NRF2 is a crucial transcription factor that regulates oxidative stress responses, and it is required for the antioxidant responses in macrophages [47]. Our results showed that the ZBTB20 knockdown attenuated the ox-LDL-induced ROS production and increased the nucleus translocation of NRF2 in the macrophages, which was significantly antagonized by the NRF2 inhibitor ML385. In addition, the ZBTB20 knockdown also rescued the ox-LDL-induced mitochondrial injury in the macrophages. Collectively, these results suggested that the ZBTB20 knockdown exerted antioxidative stress effects via enhancing the nucleus translocation of NRF2 and preventing the mitochondrial injury in macrophages. Some previous studies suggested that ZBTB20 ablation could protect mice from liver steatosis and improve hepatic lipid metabolism, the dysregulation of which may lead to AS [55]. Here, our in vivo data showed that the knockdown of ZBTB20 attenuated AS in $\mathrm{ApoE}^{-/-}$mice. Hence, it is possible to selectively target ZBTB20 using novel viral vectors, such as adenovirus and adeno-associated virus, to slow the progression of clinical immune-inflammatory diseases. However, the in vivo effects of ZBTB20 on the AS progression, as well as how ZBTB20 regulates other cell types in AS, such as endotheliocytes, still require further investigations.

Mitochondria are well known for their roles in integrating redox, efferocytosis, epigenetic, and apoptotic regulations [56]. How mitochondria function will depend on the shape and density, which are modulated by the fusion/fission balances $[57,58]$. These functions have been found to be disturbed in macrophages from the AS plaques [59]. In this study, we found that ox-LDL stimulation caused ROS production and mitochondrial injury, which were significantly attenuated by ZBTB20 silencing. Our findings were consistent with studies from Peng et al., showing that ox-LDL induced mitophagy in mitochondria [60]. DRP1 and FIS1 are two key mediators relevant to the mitochondrial fission process [61, 62], and our results showed that the ZBTB20 knockdown attenuated the ox-LDL-induced activation of DRP1/FIS1 protein. Besides, our results showed that the knockdown of ZBTB20 enhanced the nucleus translocation of NRF2 in the ox-LDL-stimulated macrophages, which was counteracted by ML385, a novel NRF2 inhibitor. Nevertheless, the NRF2 transcription factor might be one of the signaling pathways through which ZBTB20 regulated the oxidative stress level, and the deeper mechanisms remain to be explored.

Collectively, our results may imply that ZBTB20 silencing reduced oxidative stress by reducing ROS production and mitochondrial injury via NRF2 signaling. In the clinic, it may be possible to use adenovirus or adeno-associated virus targeting ZBTB20 to prevent and treat AS.

\section{Conclusions}

In conclusion, this study demonstrated that the ZBTB20 knockdown could attenuate the progression of AS, and ZBTB20 mediated ox-LDL-induced AS possibly via modulating the inflammatory responses, oxidative stress, and mitochondrial fission of macrophages.

\section{Data Availability}

All the datasets were available from the corresponding authors.

\section{Conflicts of Interest}

The authors declare no conflict of interest.

\section{Authors' Contributions}

Jun Tao, Junxiong Qiu, and Liuyi Lu contributed equally to this work.

\section{Acknowledgments}

This work was supported by the National Natural Science Foundation of China (81702618) and the Guangdong Science and Technology Department (2020B1212060018).

\section{Supplementary Materials}

Fig. S1: (A) The mRNA levels of TNF- $\alpha$ and IL- 6 after being treated with ox-LDL for $0,12,24$, and $48 \mathrm{~h}$ were determined by qRT-PCR. (B) The blank control of Figure 2(b). (C) Quantitative analysis of Western blot results in Figure 2(c). (D) Quantitative analysis of Western blot results in Figure 2(d). (E) Quantitative analysis of Western blot results in Figure 2(f). Significant differences between treatment groups were shown as ${ }^{*} P<0.05$. Fig. S2: (A) The top 15 significant GO terms and KEGG pathways from the GO categories and KEGG database. (B) The four most significant KEGG pathways analyzed with GSEA. Fig. S3: (A) Quantitative analysis of Western blot results in Figure 4(c). (B) The Western blot result of ZBTB20-siRNA knockdown efficiency. (C) The blank control of Figure 5(a). (D) Quantitative analysis of Western blot results in Figure 5(d). (E) Quantitative analysis of Western blot in Figure 5(f). Significant differences between treatment groups were shown as ${ }^{*} P<0.05$. Fig. S4: (A) The isotype controls of Figure 6(a). (B) Quantitative analysis of flow cytometry results in Figure 6(a). (C) Quantitative analysis of Western blot results in Figure 6(c). (D) The blank control of Figure 7(b). (E) Quantitative analysis of Western blot results in Figure 7(e). (F, G) Quantitative analysis of Western blot results in Figure 7(g). Significant differences between treatment groups were shown as ${ }^{*} P<0.05$. (Supplementary Materials)

\section{References}

[1] K. Kobiyama and K. Ley, "Atherosclerosis," Circulation Research., vol. 123, no. 10, pp. 1118-1120, 2018. 
[2] G. Heusch, "Coronary microvascular obstruction: the new frontier in cardioprotection," Basic Research in Cardiology, vol. 114, no. 6, p. 45, 2019.

[3] P. Libby, P. M. Ridker, and G. K. Hansson, "Progress and challenges in translating the biology of atherosclerosis," Nature, vol. 473, no. 7347, pp. 317-325, 2011.

[4] D. J. Tyrrell and D. R. Goldstein, "Ageing and atherosclerosis: vascular intrinsic and extrinsic factors and potential role of IL-6," Nature Reviews. Cardiology, vol. 18, no. 1, pp. 5868, 2021.

[5] D. A. Chistiakov, A. A. Melnichenko, V. A. Myasoedova, A. V. Grechko, and A. N. Orekhov, "Mechanisms of foam cell formation in atherosclerosis," Journal of molecular medicine (Berlin, Germany), vol. 95, no. 11, pp. 1153-1165, 2017.

[6] A. J. Kattoor, S. H. Kanuri, and J. L. Mehta, "Role of Ox-LDL and LOX-1 in atherogenesis," Current medicinal chemistry., vol. 26, no. 9, pp. 1693-1700, 2019.

[7] W. Martinet, I. Coornaert, P. Puylaert, and G. R. Y. De Meyer, "Macrophage death as a pharmacological target in atherosclerosis," Frontiers in Pharmacology, vol. 10, p. 306, 2019.

[8] A. P. West, W. Khoury-Hanold, M. Staron et al., "Mitochondrial DNA stress primes the antiviral innate immune response," Nature, vol. 520, no. 7548, pp. 553-557, 2015.

[9] E. L. Mills, B. Kelly, A. Logan et al., "Succinate dehydrogenase supports metabolic repurposing of mitochondria to drive inflammatory macrophages," Cell, vol. 167, no. 2, pp. 457470.e13, 2016.

[10] W. Peng, G. Cai, Y. Xia et al., "Mitochondrial dysfunction in atherosclerosis," DNA and cell biology., vol. 38, no. 7, pp. 597-606, 2019.

[11] Y. Chen, M. Yang, W. Huang et al., "Mitochondrial metabolic reprogramming by CD36 signaling drives macrophage inflammatory responses," Circulation Research, vol. 125, no. 12, pp. 1087-1102, 2019.

[12] Y. Wei, J. Corbalán-Campos, R. Gurung et al., "Dicer in macrophages prevents atherosclerosis by promoting mitochondrial oxidative metabolism," Circulation, vol. 138, no. 18, pp. 2007-2020, 2018.

[13] D. T. Love, C. Guo, E. I. Nikelshparg, N. A. Brazhe, O. Sosnovtseva, and C. L. Hawkins, "The role of the myeloperoxidase-derived oxidant hypothiocyanous acid (HOSCN) in the induction of mitochondrial dysfunction in macrophages," Redox Biology, vol. 36, p. 101602, 2020.

[14] T. Xin, C. Lu, J. Zhang et al., "Oxidized LDL disrupts metabolism and inhibits macrophage survival by activating a miR9/Drp1/mitochondrial fission signaling pathway," Oxidative Medicine and Cellular Longevity, vol. 2020, Article ID 8848930, 16 pages, 2020.

[15] F. Mattioli, A. Piton, B. Gérard, A. Superti-Furga, J. L. Mandel, and S. Unger, "Novel de novo mutations in ZBTB20 in Primrose syndrome with congenital hypothyroidism," American journal of medical genetics Part A., vol. 170, no. 6, pp. 16261629, 2016.

[16] M. L. Peterson, C. Ma, and B. T. Spear, "Zhx2 and Zbtb20: novel regulators of postnatal alpha-fetoprotein repression and their potential role in gene reactivation during liver cancer," Seminars in cancer biology., vol. 21, no. 1, pp. 21-27, 2011.

[17] Y. Sun, N. K. Preiss, K. B. Valenteros et al., "Zbtb20 restrains CD8 $\mathrm{T}$ cell immunometabolism and restricts memory differentiation and antitumor immunity," Journal of Immunology, vol. 205 , no. 10 , pp. 2649-2666, 2020.
[18] J. Qiu, P. Peng, M. Xin et al., “ZBTB20-mediated titanium particle-induced peri-implant osteolysis by promoting macrophage inflammatory responses," Biomaterials Science, vol. 8, no. 11, pp. 3147-3163, 2020.

[19] A. J. Ren, K. Wang, H. Zhang et al., "ZBTB20 regulates nociception and pain sensation by modulating TRP channel expression in nociceptive sensory neurons," Nature Communications, vol. 5, no. 1, p. 4984, 2014.

[20] X. Liu, P. Zhang, Y. Bao et al., "Zinc finger protein ZBTB20 promotes Toll-like receptor-triggered innate immune responses by repressing $\mathrm{I} \kappa \mathrm{B} \alpha$ gene transcription," Proceedings of the National Academy of Sciences of the United States of America, vol. 110, no. 27, pp. 11097-11102, 2013.

[21] I. Pateras, C. Giaginis, C. Tsigris, E. Patsouris, and $\mathrm{S}$. Theocharis, "NF- $\kappa \mathrm{B}$ signaling at the crossroads of inflammation and atherogenesis: searching for new therapeutic links," Expert Opinion on Therapeutic Targets, vol. 18, no. 9, pp. 1089-1101, 2014.

[22] A. Schwarz, G. A. Bonaterra, H. Schwarzbach, and R. Kinscherf, "Oxidized LDL-induced JAB1 influences NF- $\kappa$ B independent inflammatory signaling in human macrophages during foam cell formation," Journal of Biomedical Science, vol. 24, no. 1, p. 12, 2017.

[23] F. Li, Y. Yang, C. Xue et al., "Zinc finger protein ZBTB20 protects against cardiac remodelling post-myocardial infarction via ROS-TNF $\alpha /$ ASK1/JNK pathway regulation," Journal of Cellular and Molecular Medicine, vol. 24, no. 22, pp. 1338313396, 2020.

[24] M. Wagner, E. Bertero, A. Nickel et al., "Selective NADH communication from $\alpha$-ketoglutarate dehydrogenase to mitochondrial transhydrogenase prevents reactive oxygen species formation under reducing conditions in the heart," Basic Research in Cardiology, vol. 115, no. 5, p. 53, 2020.

[25] A. Vasseur, L. Cabel, O. Tredan et al., "Prognostic value of CEC count in HER2-negative metastatic breast cancer patients treated with bevacizumab and chemotherapy: a prospective validation study (UCBG COMET)," Angiogenesis, vol. 23, no. 2, pp. 193-202, 2020.

[26] J. Wang, Z. Chen, Q. Dai et al., "Intravenously delivered mesenchymal stem cells prevent microvascular obstruction formation after myocardial ischemia/reperfusion injury," Basic Research in Cardiology, vol. 115, no. 4, p. 40, 2020.

[27] D. E. Vatner, M. Oydanich, J. Zhang, D. Babici, and S. F. Vatner, "Secreted frizzled-related protein 2, a novel mechanism to induce myocardial ischemic protection through angiogenesis," Basic Research in Cardiology, vol. 115, no. 4 , p. $48,2020$.

[28] M. P. Winter, S. Sharma, J. Altmann et al., "Interruption of vascular endothelial growth factor receptor 2 signaling induces a proliferative pulmonary vasculopathy and pulmonary hypertension," Basic Research in Cardiology, vol. 115, no. 6, p. 58, 2020.

[29] Z. Radak, K. Suzuki, A. Posa, Z. Petrovszky, E. Koltai, and I. Boldogh, "The systemic role of SIRT1 in exercise mediated adaptation," Redox Biology, vol. 35, p. 101467, 2020.

[30] Y. Yang, L. Ma, C. Wang et al., "Matrix metalloproteinase-7 in platelet-activated macrophages accounts for cardiac remodeling in uremic mice," Basic Research in Cardiology, vol. 115, no. 3, p. 30, 2020.

[31] E. Bridges, H. Sheldon, E. Kleibeuker et al., "RHOQ is induced by DLL4 and regulates angiogenesis by determining the 
intracellular route of the Notch intracellular domain," Angiogenesis, vol. 23, no. 3, pp. 493-513, 2020.

[32] Y. Tan, D. Mui, S. Toan, P. Zhu, R. Li, and H. Zhou, "SERCA overexpression improves mitochondrial quality control and attenuates cardiac microvascular ischemiareperfusion injury," Mol Ther Nucleic Acids., vol. 22, pp. 696-707, 2020.

[33] T. C. Chen, J. Y. Chuang, C. Y. Ko et al., “AR ubiquitination induced by the curcumin analog suppresses growth of temozolomide-resistant glioblastoma through disrupting GPX4-mediated redox homeostasis," Redox Biology, vol. 30, p. 101413, 2020.

[34] J. Cao, X. Liu, Y. Yang et al., "Decylubiquinone suppresses breast cancer growth and metastasis by inhibiting angiogenesis via the ROS/p53/ BAI1 signaling pathway," Angiogenesis, vol. 23, no. 3, pp. 325-338, 2020.

[35] N. Abbas, F. Perbellini, and T. Thum, "Non-coding RNAs: emerging players in cardiomyocyte proliferation and cardiac regeneration," Basic Research in Cardiology, vol. 115, no. 5, p. 52, 2020.

[36] S. Deng, K. Essandoh, X. Wang et al., "Tsg101 positively regulates P62-Keap1-Nrf2 pathway to protect hearts against oxidative damage," Redox Biology, vol. 32, p. 101453, 2020.

[37] K. Fukada and K. Kajiya, "Age-related structural alterations of skeletal muscles and associated capillaries," Angiogenesis, vol. 23, no. 2, pp. 79-82, 2020.

[38] X. Lu, Y. He, C. Tang et al., "Triad3A attenuates pathological cardiac hypertrophy involving the augmentation of ubiquitination-mediated degradation of TLR4 and TLR9," Basic Research in Cardiology, vol. 115, no. 2, p. 19, 2020.

[39] R. Khan, V. Spagnoli, J. C. Tardif, and P. L. L'Allier, "Novel anti-inflammatory therapies for the treatment of atherosclerosis," Atherosclerosis, vol. 240, no. 2, pp. 497-509, 2015.

[40] C. Khatana, N. K. Saini, S. Chakrabarti et al., "Mechanistic insights into the oxidized low-density lipoprotein-induced atherosclerosis," Oxidative Medicine and Cellular Longevity, vol. 2020, Article ID 5245308, 14 pages, 2020.

[41] M. Kurano, N. Iso-O, M. Hara et al., "Plant sterols increased IL-6 and TNF- $\alpha$ secretion from macrophages, but to a lesser extent than cholesterol," Journal of atherosclerosis and thrombosis., vol. 18, no. 5, pp. 373-383, 2011.

[42] N. Haddy, C. Sass, S. Droesch et al., "IL-6, TNF- $\alpha$ and atherosclerosis risk indicators in a healthy family population: the STANISLAS cohort," Atherosclerosis, vol. 170, no. 2, pp. 277-283, 2003.

[43] M. P. de Winther, E. Kanters, G. Kraal, and M. H. Hofker, "Nuclear factor $\kappa \mathrm{B}$ signaling in atherogenesis," Arteriosclerosis, thrombosis, and vascular biology., vol. 25, no. 5, pp. 904-914, 2005.

[44] S. Li, J. Qiu, L. Qin et al., "NOD2 negatively regulated titanium particle-induced osteolysis in mice," Biomaterials Science, vol. 7, no. 7, pp. 2702-2715, 2019.

[45] L. Zhang, L. Lu, X. Zhong et al., "Metformin reduced NLRP3 inflammasome activity in Ox-LDL stimulated macrophages through adenosine monophosphate activated protein kinase and protein phosphatase 2A," European journal of pharmacology., vol. 852, pp. 99-106, 2019.

[46] X. Cao, N. Zhu, L. Li et al., "Y-box binding protein 1 regulates ox-LDL mediated inflammatory responses and lipid uptake in macrophages," Free radical biology \& medicine., vol. 141, pp. 10-20, 2019.
[47] Y. Zhang, X. Zhou, M. Zhang, L. Cheng, Y. Zhang, and $\mathrm{X}$. Wang, "ZBTB20 promotes cell migration and invasion of gastric cancer by inhibiting $\mathrm{I} \kappa \mathrm{B} \alpha$ to induce NF- $\kappa \mathrm{B}$ activation," Artif Cells Nanomed Biotechnol., vol. 47, no. 1, pp. 3862-3872, 2019.

[48] S. Gu, S. Ran, F. Qin et al., "Human dental pulp stem cells via the NF- $\kappa \mathrm{B}$ pathway," Cellular Physiology and Biochemistry, vol. 36, no. 5, pp. 1725-1734, 2015.

[49] K. Taketa, T. Matsumura, M. Yano et al., "Oxidized low density lipoprotein activates peroxisome proliferatoractivated receptor- $\alpha(\operatorname{PPAR} \alpha)$ and PPAR $\gamma$ through MAPKdependent COX-2 expression in macrophages*," The Journal of Biological Chemistry, vol. 283, no. 15, pp. $9852-$ 9862, 2008.

[50] Y. Bi, J. Chen, F. Hu, J. Liu, M. Li, and L. Zhao, "M2 macrophages as a potential target for antiatherosclerosis treatment," Neural plasticity., vol. 2019, article 6724903, 21 pages, 2019.

[51] J. Liao, X. An, X. Yang et al., "Deficiency of LMP10 attenuates diet-induced atherosclerosis by inhibiting macrophage polarization and inflammation in apolipoprotein E deficient mice," Frontiers in Cell and Development Biology, vol. 8, p. 592048, 2020.

[52] D. A. Chistiakov, V. A. Myasoedova, V. V. Revin, A. N. Orekhov, and Y. V. Bobryshev, "The impact of interferonregulatory factors to macrophage differentiation and polarization into M1 and M2," Immunobiology, vol. 223, no. 1, pp. 101-111, 2018.

[53] L. Håversen, J. P. Sundelin, A. Mardinoglu et al., "Vimentin deficiency in macrophages induces increased oxidative stress and vascular inflammation but attenuates atherosclerosis in mice," Scientific Reports, vol. 8, no. 1, p. 16973, 2018.

[54] D. D. Chen, L. L. Hui, X. C. Zhang, and Q. Chang, "NEAT1 contributes to ox-LDL-induced inflammation and oxidative stress in macrophages through inhibiting miR128," Journal of Cellular Biochemistry, vol. 120, no. 2, pp. 2493-2501, 2018.

[55] G. Liu, L. Zhou, H. Zhang et al., "Regulation of hepatic lipogenesis by the zinc finger protein Zbtb20," Nature Communications, vol. 8, no. 1, p. 14824, 2017.

[56] A. Shteinfer-Kuzmine, A. Verma, T. Arif, O. Aizenberg, A. Paul, and V. Shoshan-Barmaz, "Mitochondria and nucleus cross-talk: signaling in metabolism, apoptosis, and differentiation, and function in cancer," IUBMB Life, 2020.

[57] H. Xian and Y. C. Liou, "Functions of outer mitochondrial membrane proteins: mediating the crosstalk between mitochondrial dynamics and mitophagy," Cell Death and Differentiation, 2020.

[58] J. Wang, S. Toan, and H. Zhou, "Mitochondrial quality control in cardiac microvascular ischemia-reperfusion injury: new insights into the mechanisms and therapeutic potentials," Pharmacological Research, vol. 156, p. 104771, 2020.

[59] A. Dumont, M. Lee, T. Barouillet, A. Murphy, and L. YvanCharvet, "Mitochondria orchestrate macrophage effector functions in atherosclerosis," Molecular aspects of medicine., p. 100922, 2020.

[60] X. Peng, H. Chen, Y. Li, D. Huang, B. Huang, and D. Sun, "Effects of NIX-mediated mitophagy on ox-LDL-induced macrophage pyroptosis in atherosclerosis," Cell biology international., vol. 44, no. 7, pp. 1481-1490, 2020. 
[61] A. U. Joshi, N. L. Saw, M. Shamloo, and D. Mochly-Rosen, "Drp1/Fis1 interaction mediates mitochondrial dysfunction, bioenergetic failure and cognitive decline in Alzheimer's disease," Oncotarget, vol. 9, no. 5, pp. 6128-6143, 2018.

[62] J. Wang and H. Zhou, "Mitochondrial quality control mechanisms as molecular targets in cardiac ischemia $* *-* *$ reperfusion injury," Acta Pharmaceutica Sinica B, vol. 10, no. 10, pp. 1866-1879, 2020. 